TRANSACTIONS OF THE

AMERICAN MATHEMATICAL SOCIETY

Volume 315, Number 2, October 1989

\title{
ON INFINITE ROOT SYSTEMS
}

\author{
R. V. MOODY AND A. PIANZOLA \\ Dedicated to our good friend Stephen Berman
}

\begin{abstract}
We define in an axiomatic fashion the concept of a set of root data that generalizes the usual concept of root system of a Kac-Moody Lie algebra. We study these objects from a purely formal and geometrical point of view as well as in relation to their associated Lie algebras. This leads to a coherent theory of root systems, bases, subroot systems, Lie algebras defined by root data, and subalgebras.
\end{abstract}

\section{INTRODUCTION}

The theory of finite root systems is very well understood and has been the subject of several beautiful expositions [Bbk, Stn]. In comparison, the theory of infinite root systems is still somewhat awkward and a number of difficulties remain to be overcome. Among the most obvious of these is the problem of subroot systems. We begin by illustrating this with an example.

Let $A$ be a (generalized) Cartan matrix $A=\left(A_{i j}\right)_{1 \leq i, j \leq l}$. By definition $A$ satisfies the axioms

For all $i \leq i, j \leq l$,

$$
\begin{aligned}
& A_{i j} \in \mathbf{Z}, \\
& A_{i i}=2, \\
& A_{i j} \leq 0 \text { whenever } i \neq j, \\
& A_{i j}=0 \Leftrightarrow A_{j i}=0 .
\end{aligned}
$$

A realization of $A$ (over a field $\mathbf{K}$ of characteristic 0 ) consists of a finitedimensional vector space $V$ and a pair of sets $\Pi=\left\{\alpha_{1}, \ldots, \alpha_{l}\right\} \subset V, \Pi^{\vee}=$ $\left\{\alpha_{1}^{\vee}, \ldots, \alpha_{l}^{\vee}\right\} \subset V^{*} \quad\left(V^{*}=\right.$ dual space of $\left.V\right)$ such that

(a) the elements of $\Pi$ (respectively $\Pi^{\vee}$ ) are linearly independent in $V$ (respectively $V^{*}$ )

(b) $\left\langle\alpha_{i}, \alpha_{j}^{\vee}\right\rangle=A_{i j}$ for all $1 \leq i, j \leq l$ where $\langle\cdot, \cdot\rangle$ denotes the natural pairing of $V \times V^{*} \rightarrow \mathbf{K}$.

Received by the editors March 9, 1988.

1980 Mathematics Subject Classification (1985 Revision). Primary 17B67; Secondary 81G20.

Both authors acknowledge the continuous support of the Natural Sciences and Engineering Research Council of Canada. 
Given a realization $R=\left(V, \Pi, \Pi^{\vee}\right)$ of $A$ we can define the Weyl group $W$ (respectively dual Weyl group $W^{\vee}$ ) as the group generated by the reflections $r_{i}$ (respectively $r_{i}^{\vee}$ ) where

$$
\begin{gathered}
r_{i}: v \mapsto v-\left\langle v, \alpha_{i}^{\vee}\right\rangle \alpha_{i} \quad \forall v \in V, \\
r_{i}^{\vee}: v^{\vee} \mapsto v^{\vee}-\left\langle\alpha_{i}, v^{\vee}\right\rangle \alpha_{i}^{\vee} \quad \forall v^{\vee} \in V^{\vee} .
\end{gathered}
$$

Since $W \simeq W^{\vee}$ via $r_{i} \leftrightarrow r_{i}^{\vee}$ we normally identify these two groups. Under this identification

$$
\left\langle w v, v^{\vee}\right\rangle=\left\langle v, w^{-1} v^{\vee}\right\rangle \quad \text { for all } v \in V, v^{\vee} \in V^{\vee} \text { and for all } w \in W .
$$

The (real) root system of $R$ is now defined as

$$
{ }^{\mathrm{re}} \Delta:=W \Pi
$$

and the corresponding (real) coroot system as

$$
{ }^{\mathrm{re}} \Delta^{\vee}:=W \Pi^{\vee} \text {. }
$$

We say "real" because the Kac-Moody Lie algebra $\mathfrak{g}(A)$ associated to $A$ has roots outside ${ }^{\mathrm{re}} \Delta$, these additional roots being the set of imaginary roots ${ }^{\mathrm{im}} \Delta$. (By convention here 0 is a root so $0 \in{ }^{\mathrm{im}} \Delta$ always.) For the moment let us consider only the real roots.

For each $\alpha \in{ }^{\mathrm{re}} \Delta$, writing $\alpha=w \alpha_{i}, w \in W, \alpha_{i} \in \Pi$, we define a reflection $r_{\alpha}=w r_{i} w^{-1}$ and a coroot $\alpha^{\vee}=w \alpha_{i}^{\vee}$. Both $r_{\alpha}$ and $\alpha^{\vee}$ depend only on $\alpha$ (not on the choice of $w$ and $\alpha_{i}$ ) and

$$
\begin{aligned}
& r_{\alpha}: v \mapsto v-\left\langle v, \alpha^{\vee}\right\rangle \alpha \quad \forall v \in V, \\
& v^{\vee} \mapsto v^{\vee}-\left\langle\alpha, v^{\vee}\right\rangle \alpha^{\vee} \quad \forall v^{\vee} \in V^{\vee} .
\end{aligned}
$$

Now we can define subroot systems. A nonempty subset $\Omega$ of ${ }^{\mathrm{re}} \Delta$ is a subroot system if for all $\alpha$ and $\beta$ in ${ }^{\mathrm{re}} \Delta$

$$
\alpha, \beta \in \Omega \Rightarrow r_{\alpha} \beta \in \Omega \text {. }
$$

To justify this definition (which is the natural one in view of the finite-dimensional theory) we need to produce a Cartan matrix and a base (like $\Pi$ ) for $\Omega$. The following example illustrates that this is not always possible within the framework we have here.

Example 1 (Maxwell's Demon). Let

$$
A=\left(\begin{array}{rrr}
2 & -2 & 0 \\
-2 & 2 & -2 \\
0 & -2 & 2
\end{array}\right) \text {. }
$$

Let $V=\mathbf{R} \alpha_{0} \oplus \mathbf{R} \alpha_{1} \oplus \mathbf{R} \alpha_{2}$ be a 3-dimensional real space, $V^{*}$ its dual, and $\langle\cdot, \cdot\rangle: V \times V^{*} \rightarrow \mathbf{R}$ the natural pairing. Let $\Pi=\left\{\alpha_{0}, \alpha_{1}, \alpha_{2}\right\} \subset V$ and define $\Pi^{\vee}=\left\{\alpha_{0}^{\vee}, \alpha_{1}^{\vee}, \alpha_{2}^{\vee}\right\} \subset V^{*}$ by $\left\langle\alpha_{i}, \alpha_{j}^{\vee}\right\rangle=A_{i j} ; 0 \leq i, j \leq 2$. With the Weyl 
group $W=\left\langle r_{i} \mid i=0,1,2\right\rangle$ defined in the usual fashion and identifying $W^{\vee}$ with $W$ let

$$
\beta_{k}=\left(r_{2} r_{1}\right)^{k} \alpha_{0} \text { and } \beta_{k}^{\vee}=\left(r_{2} r_{1}\right)^{k} \alpha_{0}^{\vee} \text {. }
$$

Claim 1. $\beta_{k}=\alpha_{0}+2 k^{2} \alpha_{1}+2\left(k^{2}+k\right) \alpha_{2}$ and $\beta_{k}^{\vee}=\alpha_{0}+2 k^{2} \alpha_{1}^{\vee}+2\left(k^{2}+k\right) \alpha_{2}^{\vee}$ for all $k \in \mathbf{Z}$.

To establish this, reason by induction on $k$.

Claim 2. For all $p, q \in \mathbf{Z}, p \neq q$ we have

$$
\left\langle\beta_{p}, \beta_{q}^{\vee}\right\rangle<0 \text { and }\left\langle\beta_{p}, \beta_{p}^{\vee}\right\rangle=2 \text {. }
$$

Assume without loss of generality that $q-p=k \geq 0$,

$$
\begin{aligned}
\left\langle\beta_{p}, \beta_{q}^{\vee}\right\rangle & =\left\langle\left(r_{2} r_{1}\right)^{p} \alpha_{0},\left(r_{2} r_{1}\right)^{q} \alpha_{0}^{\vee}\right\rangle \\
& =\left\langle\alpha_{0},\left(r_{2} r_{1}\right)^{k} \alpha_{0}^{\vee}\right\rangle \\
& =\left\langle\alpha_{0}, \alpha_{0}^{\vee}+2 k^{2} \alpha_{1}^{\vee}+2\left(k^{2}+k\right) \alpha_{2}^{\vee}\right\rangle \\
& =2-4 k^{2} .
\end{aligned}
$$

We return to our main construction. Let

$$
B=\left(\left\langle\beta_{i}, \beta_{j}^{\vee}\right\rangle\right)_{i, j \in \mathbf{Z}} .
$$

Then $B$ is a Cartan matrix (infinite of rank 3 ). Let

and define

$$
\Upsilon=\left\{\beta_{k}\right\}_{k \in \mathbf{Z}} \text { and } \Upsilon^{\vee}=\left\{\beta_{k}^{\vee}\right\}_{k \in \mathbf{Z}}
$$

where

$$
\Omega=W_{\Upsilon} \Upsilon \quad \text { and } \quad \Omega^{\vee}=W_{\Upsilon} \Upsilon^{\vee}
$$

$$
W_{\Upsilon}=\left\langle r_{\beta_{k}} \mid k \in \mathbf{Z}\right\rangle \subset W .
$$

It is clear that $\Omega$ is a subroot system of $\Sigma:=W \Pi$. However, $\Omega$ does not admit a base in the sense that $\Sigma$ does, as we will see in the sequel.

There is no shortage of examples of this type. For instance, one can let $k$ above vary over any infinite subset of $\mathbf{Z}$ and arrive at a similar situation. This shows that any theory of infinite root systems must allow the possibility of infinite bases and that the linear independence condition, which is usually assumed for bases, is too strong.

In this paper we give a modified set of axioms which allow us to address five problems which seem to us to be essential.

(a) The theory should be rational in the sense that it allows shifting of base fields.

(b) Subroot systems should satisfy the same axioms as root systems.

(c) To these new root systems we should be able to associate Lie algebras and (closed) subroot systems should correspond to subalgebras.

(d) There should be a conjugacy theorem for bases.

(e) The theory should lead to a chamber geometry in the style of [Bbk]. 
The chamber geometry in fact is the main tool that we use in the study of conjugacy and subroot problems. The rationality properties of root systems allow us to work over $\mathbf{R}$. Among the root systems that are included in our treatment is the root system of the Lorentzian lattice $\Lambda^{25,1}$ in 26-dimensional space. Our work has obvious similarities to that of Vinberg [Vnb], who also studied chambers with infinitely many faces and infinitely many symmetries. His work differs from ours in the important respect that he considers only discrete groups in hyperbolic space. Although the Demon is realized in hyperbolic space, this is not so for root systems in general.

We would like to thank D. Maclean for his computer-drawn picture of the Demon appearing in $\S 5$.

\section{Root DATA}

Let $\mathbf{K}$ be a field of characteristic 0 . By a set of root data over $\mathbf{K}$ we will understand a 6-tuple

$$
\mathscr{D}=\left(A, \Pi, \Pi^{\vee}, V, V^{\vee},\langle\cdot, \cdot\rangle\right)
$$

consisting of

(RD1) a Cartan matrix $A=\left(A_{i j}\right)_{i, j \in \mathbf{J}}$ where $\mathbf{J}$ is an index set;

(RD2) a pair of vector spaces $V$ and $V^{\vee}$ over $\mathbf{K}$ together with a nondegenerate pairing $\langle\cdot, \cdot\rangle: V \times V^{\vee} \rightarrow \mathbf{K}$;

(RD3) subsets

$$
\Pi=\left\{\alpha_{j}\right\}_{j \in \mathbf{J}} \subset V, \quad \Pi^{\vee}=\left\{\alpha_{j}^{\vee}\right\}_{j \in \mathbf{J}} \subset V^{\vee}
$$

such that $\left\langle\alpha_{i}, \alpha_{j}^{\vee}\right\rangle=A_{i j}$ for all $i, j \in \mathbf{J}$.

Furthermore we require

(RD4) $Q:=\sum_{j \in \mathbf{J}} \mathbf{Z} \alpha_{j}$ and $Q^{\vee}:=\sum_{j \in \mathbf{J}} \mathbf{Z} \alpha_{j}^{\vee}$ are free abelian groups admitting bases of the form $\left\{\gamma_{i}\right\}_{i \in \mathbf{I}} \subset Q$ and $\left\{\gamma_{i}^{\vee}\right\}_{i \in \mathbf{I}} \subset Q^{\vee}$ where $\mathbf{I}$ is an index set and where the $\gamma_{i}$ and $\gamma_{i}^{\vee}$ are linearly independent over $\mathbf{K}$ and satisfy

$$
\Pi \subset \bigoplus_{i \in \mathbf{I}} \mathbf{N} \gamma_{i} \text { and } \Pi^{\vee} \subset \bigoplus_{i \in \mathbf{I}} \mathbf{N} \gamma_{i}^{\vee} .
$$

The last of these axioms requires some comment. The root system of a KacMoody Lie algebra $\mathfrak{g}$ over $\mathbf{K}$ is a rational geometric object to the extent that $\mathfrak{g}$ can be obtained from a rational Kac-Moody Lie algebra by extension of the base field. Axiom RD4 can be motivated by this fact and we shall see later $(\S 4)$ that sets of root data can always be obtained from rational sets of root data.

The assumption that $Q$ and $Q^{\vee}$ are lattices parallels the analogous fact for Kac-Moody Lie algebras and Lie algebras with triangular decompositions [MP]. Note, however, that $\Pi$ and $\Pi^{\vee}$ are not assumed to be bases of these lattices even though $\Pi$ and $\Pi^{\vee}$ lie each entirely in one orthant of a lattice. This last fact is analogous to the division of roots into positive and negative and will allow us to build the geometry of this new type of root system. 
Example 2. If $A$ is a Cartan matrix and $R=\left(V, \Pi, \Pi^{\vee}\right)$ is a realization of $A$ then $\left(A, \Pi, \Pi^{\vee}, V, V^{*},\langle\cdot, \cdot\rangle\right)$ is a set of root data.

Example 3. Let the notation be that of the Maxwell Demon as in Example 1. Then $\mathscr{D}=\left(B, \Upsilon, \Upsilon^{\vee}, V, V^{\vee},\langle\cdot, \cdot\rangle\right)$ is a set of root data. Indeed, to satisfy RD4 we may simply take

$$
\gamma_{0}=\alpha_{0}, \quad \gamma_{1}=2 \alpha_{1}, \quad \gamma_{2}=4 \alpha_{2}
$$

and

$$
\gamma_{0}^{\vee}=\alpha_{0}^{\vee}, \quad \gamma_{1}^{\vee}=2 \alpha_{1}^{\vee}, \quad \gamma_{2}^{\vee}=4 \alpha_{2}^{\vee} .
$$

The reader can verify that $\sum_{i \in \mathbf{Z}} \mathbf{Z} \beta_{i}=\bigoplus_{i=0}^{2} \mathbf{Z} \gamma_{i}$ and $\sum_{i \in \mathbf{Z}} \mathbf{Z} \beta_{i}^{\vee}=\bigoplus_{i=0}^{2} \mathbf{Z} \gamma_{i}^{\vee}$.

Example 4 (Monstrous root data). Let $\Lambda^{25,1}$ be the unimodular lattice of signature $\left(1^{25},-1\right)$ in 26-dimensional space $\mathbf{R}^{25,1}$ with symmetric bilinear form $(\cdot \mid \cdot)$ defined by

$$
(x \mid x)=\sum_{i=0}^{24} x_{i}^{2}-x_{25}^{2}, \quad x=\left(x_{0}, \ldots, x_{24} ; x_{25}\right) .
$$

Let $\Pi$ be the set of Leech roots in $\Lambda^{25,1}$ and let $\alpha^{\vee}=\alpha$ for all $\alpha \in \Pi$. Then $L:=((\alpha \mid \beta))_{\alpha, \beta \in \Pi}$ is a Cartan matrix and

$$
\mathscr{M}:=\langle L, \Pi, \Pi, V, V,(\cdot \mid \cdot)\rangle
$$

is a set of root data for $L$. Later we will see that the associated Lie algebra $\mathfrak{g}(\mathscr{M})$ is the Monster Lie algebra of Borcherds, Conway, Queen, and Sloane [CS]. See $\S 8$ for details.

The linear independence of $\Pi$ and $\Pi^{\vee}$ in the Kac-Moody setting is replaced in the root data situation by the following weak independence property.

Proposition 1. Let $\mathscr{D}$ be a set of root data as above. Then for all $k \in \mathbf{J}$

$$
\left\{\begin{array}{l}
\mathbf{Z} \alpha_{k} \cap\left(\sum_{j \neq k} \mathbf{N} \alpha_{j}\right)=(0), \\
\mathbf{Z} \alpha_{k}^{\vee} \cap\left(\sum_{j \neq k} \mathbf{N} \alpha_{j}^{\vee}\right)=(0) .
\end{array}\right.
$$

Proof. Suppose $-n_{k} \alpha_{k}=\sum_{j \neq k} n_{j} \alpha_{j}$ where $n_{j} \geq 0$ for all $j \neq k$. If $n_{k}<0$ by computing $\left\langle\cdot, \alpha_{k}^{\vee}\right\rangle$ on both sides we obtain a contradiction. If $n_{k}>0$, then

$$
\sum_{j \in \mathrm{J}} n_{j} \alpha_{j}=0
$$

where $n_{j} \geq 0$ for all $j \in \mathbf{J}$ and not all $n_{j}=0$. By RD4 we can write

$$
\alpha_{j}=\sum_{i \in \mathbf{I}} c_{i j} \gamma_{i}
$$


with $c_{i j} \geq 0$. Combining $(*)$ and $(* *)$ then gives a contradiction. The case of $\Pi^{\vee}$ is similar and WIP then follows.

Given a set of root data $\mathscr{D}$ we define $W \subset \mathrm{GL}(V)$ and $W^{\vee} \subset \mathrm{GL}\left(V^{\vee}\right)$ in the usual fashion: for each $j \in \mathbf{J}, r_{j} \in \mathbf{G L}(V)$ and $r_{j}^{\vee} \in \mathrm{GL}\left(V^{\vee}\right)$ are defined by

$$
\begin{array}{cl}
r_{\alpha_{j}}:=r_{j}: \alpha \mapsto \alpha-\left\langle\alpha, \alpha_{j}^{\vee}\right\rangle \alpha_{j} & \text { for all } \alpha \in V, \\
r_{\alpha_{j}^{\vee}}:=r_{j}^{\vee}: h \mapsto h-\left\langle\alpha_{j}, h\right\rangle \alpha_{j}^{\vee} & \text { for all } h \in V^{\vee} .
\end{array}
$$

Then

$$
W:=\left\langle r_{j} \mid j \in \mathbf{J}\right\rangle, \quad W^{\vee}:=\left\langle r_{j}^{\vee} \mid j \in \mathbf{J}\right\rangle
$$

and the systems of real roots and coroots are defined by

$$
\Sigma:=W \Pi, \quad \Sigma^{\vee}:=W^{\vee} \Pi^{\vee} .
$$

We also call $\Sigma$ and $\Sigma^{\vee}$ the root and coroot systems of $\mathscr{D}$.

We also define the usual accountrements

$$
\begin{gathered}
\Sigma_{+}:=\left(\sum_{j \in \mathbf{J}} \mathbb{N} \alpha_{j}\right) \cap \Sigma, \\
\Sigma_{+}^{\vee}:=\left(\sum_{j \in \mathbf{J}} \mathbb{N} \alpha_{j}^{\vee}\right) \cap \Sigma, \\
\Sigma_{-}:=-\Sigma_{+} \quad \text { and } \quad \Sigma_{-}^{\vee}:=-\Sigma_{+}^{\vee}, \\
Q:=\sum_{j \in \mathbf{J}} \mathbf{Z}_{j}, \quad Q_{\mathbf{K}}:=\sum_{j \in \mathbf{J}} \mathbf{K} \alpha_{j}, \\
Q^{\vee}:=\sum_{j \in \mathbf{J}} \mathbf{Z}_{\alpha_{j}^{\vee}}^{\vee}, \quad Q_{\mathbf{K}}^{\vee}:=\sum_{j \in \mathbf{J}} \mathbf{K} \alpha_{j}^{\vee} .
\end{gathered}
$$

We proceed now to outline the basic expected results that follow from the definition.

Proposition 2. There exists a unique group isomorphism ${ }^{*}: W \rightarrow W^{\vee}$ satisfying $r_{j}^{*}=r_{j}^{\vee}$. Moreover, if we identify $W$ and $W^{\vee}$ via this isomorphism then

$$
\langle\alpha, h\rangle=\langle w \alpha, w h\rangle
$$

for all $\alpha \in V, h \in V^{\vee}$, and $w \in W$.

Proof. This uses only the definition of $r_{i}$ and $r_{i}^{\vee}$ and the nondegeneracy of $\langle\cdot, \cdot\rangle$.

Henceforth $W$ and $W^{\vee}$ will be identified as in Proposition 2.

Given any Cartan matrix $A=\left(A_{i j}\right)_{i, j \in \mathbf{J}}$ we can form a realization of $A$ [Kac, MP]. By definition this consists of a vector space $\widetilde{V}$ and subsets of linearly independent elements $\widetilde{\Pi}=\left\{\tilde{\alpha}_{i}\right\}_{i \in \mathrm{J}} \subset V, \widetilde{\Pi}^{\vee}=\left\{\tilde{\alpha}_{i}^{\vee}\right\}_{i \in \mathrm{J}} \subset \widetilde{V}^{*}$ such that 
$\left\langle\tilde{\alpha}_{i}, \tilde{\alpha}_{j}^{\vee}\right\rangle=A_{i j}$. This immediately gives rise to a set of root data $\widetilde{D}$ with Cartan matrix $A$. For RD4 we can set $\mathbf{I}=\mathbf{J}$ and $\left\{\gamma_{i}\right\}_{i \in \mathbf{I}}=\widetilde{\Pi}$ and $\left\{\gamma_{i}^{\vee}\right\}_{i \in \mathbf{I}}=\widetilde{\Pi}^{\vee}$.

Given a realization of $A$ we can construct a Lie algebra $\mathfrak{g}(A)$ from $A$ whose Cartan subalgebra is $\widetilde{V}^{*}$ and whose set $\widetilde{\Sigma}$ of real roots (respectively coroots) is $\widetilde{W} \widetilde{\Pi}$ (respectively $\widetilde{W} \widetilde{\Pi}^{\vee}$ ). Thus in the case of a realization we can identify the set of real roots (coroots) with the set of real roots (coroots) of a Kac-Moody algebra. In particular,

$$
\widetilde{\Sigma}=\tilde{\Sigma}_{+} \cup \widetilde{\Sigma}_{-}, \quad \widetilde{\Sigma}^{\vee}=\widetilde{\Sigma}_{+} \cup \widetilde{\Sigma}_{-}
$$

(both unions disjoint).

It is possible to prove that this partition into positive and negative parts exists without appeal to the Lie algebra (see [Ljg] and [MP]). In any case, we will later see that this positive-negative duality also holds for arbitrary sets of root data $\mathscr{D}=\left(A, V, V^{\vee}, \Pi, \Pi^{\vee},\langle\cdot, \cdot\rangle\right)$.

Let $\mathscr{D}$ be a set of root data and let $\widetilde{\mathscr{D}}=\left(A, \widetilde{V}, \widetilde{V}^{*}, \widetilde{\Pi}, \widetilde{\Pi}^{\vee},\langle\cdot, \cdot\rangle\right)$ be the set of root data obtained by means of a realization of $A$ as we have described above. A set of root data $\widetilde{\mathscr{D}}$ of this form will be called the universal covering of $\mathscr{D}$. All the objects naturally associated with $\widetilde{\mathscr{D}}$ carry tildes. There are unique linear maps

satisfying

$$
\psi: \widetilde{Q}_{\mathbf{K}} \rightarrow Q_{\mathbf{K}}, \quad \psi^{\vee}: \widetilde{Q}_{\mathbf{K}}^{\vee} \rightarrow Q_{\mathbf{K}}^{\vee}
$$

$$
\psi\left(\tilde{\alpha}_{i}\right)=\alpha_{i}, \quad \psi^{\vee}\left(\tilde{\alpha}_{i}^{\vee}\right)=\alpha_{i}^{\vee} \quad \text { for all } i \in \mathbf{J} .
$$

Proposition 3. Let the notation be as above.

(i) The mapping $r_{\tilde{\alpha}_{i}} \mapsto r_{\alpha_{i}}$ extends uniquely to a group isomorphism $\sigma$ : $\widetilde{W} \rightarrow W$.

(ii) Relative to $\sigma, \psi$ is a $(\widetilde{W}, W)$-equivariant map; i.e., for all $\tilde{w} \in \widetilde{W}$ the diagram

$$
\begin{array}{cc}
\tilde{Q}_{\mathbf{K}} \stackrel{\psi}{\longrightarrow} Q_{\mathbf{K}} \\
\tilde{w} \downarrow & \sigma(\tilde{w}) \downarrow \\
\tilde{Q}_{\mathbf{K}} \stackrel{\psi}{\longrightarrow} Q_{\mathbf{K}}
\end{array}
$$

commutes. Likewise $\psi^{\vee}$ is $(\widetilde{W}, W)$-equivariant.

(iii) For all $\tilde{v} \in \tilde{Q}_{\mathbf{K}}, \tilde{v}^{\vee} \in \tilde{Q}_{\mathbf{K}}^{\vee}$

$$
\left\langle\tilde{v}, \tilde{v}^{\vee}\right\rangle=\left\langle\psi \tilde{v}, \psi^{\vee} \tilde{v}^{\vee}\right\rangle \text {. }
$$

(iv) $\psi: \widetilde{\Sigma} \rightarrow \Sigma$ and $\psi^{\vee}: \widetilde{\Sigma}^{\vee} \rightarrow \Sigma^{\vee}$ are bijections.

(v) $\operatorname{ker} \psi=\left\{\tilde{\beta} \in \tilde{Q}_{\mathbf{K}} \mid\left\langle\tilde{\beta}, \tilde{\alpha}_{j}^{\vee}\right\rangle=0 \quad \forall j \in \mathbf{J}\right\}$.

In particular, if $A$ has linearly independent rows then $\psi$ is injective. Similar statements hold for $\psi^{\vee}$ ( use rows of $A^{T}$ ).

Proof. If the homomorphism $\sigma$ exists it is clearly unique. Let $i, j \in \mathbf{J}$. Then

$$
r_{\tilde{\alpha}_{j}}: \tilde{\alpha}_{i} \mapsto \tilde{\alpha}_{i}-\left\langle\tilde{\alpha}_{i}, \tilde{\alpha}_{j}^{\vee}\right\rangle \tilde{\alpha}_{j}=\tilde{\alpha}_{i}-A_{i j} \tilde{\alpha}_{j}
$$


while

This shows that for all $j_{1}, \ldots, j_{n} \in \mathbf{J}$

$$
r_{\alpha_{j}}: \alpha_{i} \mapsto \alpha_{i}-A_{i j} \alpha_{j}
$$

$$
r_{\tilde{\alpha}_{j_{1}}} \cdots r_{\tilde{\alpha}_{j_{n}}} \tilde{\alpha}_{i}=\sum_{j \in \mathbf{J}} c_{i j} \tilde{\alpha}_{j} \Rightarrow r_{\alpha_{j_{1}}} \cdots r_{\alpha_{j_{n}}} \alpha_{i}=\sum_{j \in \mathbf{J}} c_{i j} \alpha_{j} .
$$

(The reverse implication is not immediate because the $c_{i j}$ on the right-hand side may not be unique.) From $(*)$ it is easy to conclude that $\sigma$ exists and that the diagram of (ii) commutes.

Suppose that $\tilde{w} \in \widetilde{W}$ and $\sigma(\tilde{w})=1$. Then for each $i, \psi\left(\tilde{w} \tilde{\alpha}_{i}\right)=\alpha_{i}$. Writing $\tilde{w} \tilde{\alpha}_{i}=\sum c_{j} \tilde{\alpha}_{j}$ where all $c_{j}$ have the same sign we obtain $\sum c_{j} \alpha_{j}=\alpha_{i}$. This contradicts WIP unless $c_{i}=1$ and $c_{j}=0$ for $j \neq i$. Thus $\tilde{w} \tilde{\alpha}_{i}=\tilde{\alpha}_{i}$ for all $i$. But this is well known to imply $\tilde{w}=1$ [MP].

(iii) This is obvious.

(iv) Suppose $\tilde{\alpha}, \tilde{\beta} \subset \widetilde{\Sigma}, \tilde{\alpha} \neq \tilde{\beta}$, and $\psi \tilde{\alpha}=\psi \tilde{\beta}$. By (ii) it follows that $\psi \tilde{\alpha}_{j}=\psi \tilde{\gamma}$ for some $\tilde{\gamma} \neq \tilde{\alpha}_{j}$ and some $j \in \mathbf{J}$. Now $\psi \tilde{\gamma} \in \Sigma_{+}$or $\psi \tilde{\gamma} \in \Sigma_{-}$and in either case $\alpha_{j}=\psi \tilde{\gamma}$ will contradict WIP. Having established that the restriction of $\psi$ to $\widetilde{\Sigma}$ is injective, we use the fact that

$$
\psi \widetilde{\Sigma}=\psi \widetilde{W} \widetilde{\Pi}=W \psi \widetilde{\Pi}=W \Pi=\Sigma
$$

to conclude that $\psi$ is a bijection of $\widetilde{\Sigma}$ onto $\Sigma$.

(v) This is a simple calculation.

Corollary. In a set of root data the following wonderful union property holds

$$
\Sigma=\Sigma_{+} \cup \Sigma_{-} \text {and } \Sigma^{\vee}=\Sigma_{+}^{\vee} \cup \Sigma_{-}^{\vee}
$$

( the unions being disjoint) .

Proof. $\Sigma=\psi \widetilde{\Sigma}=\psi\left(\widetilde{\Sigma}_{+} \cup \widetilde{\Sigma}_{-}\right) \subset \Sigma_{+} \cup \Sigma_{-}$, which shows that $\Sigma=\Sigma_{+} \cup \Sigma_{-}$. That $\Sigma_{+} \cap \Sigma_{-}=(0)$ follows from WIP.

Given $\alpha \in \Sigma$ there is a unique $\tilde{\alpha} \in \widetilde{\Sigma}$ such that $\psi \tilde{\alpha}=\alpha$ (Proposition 3(iv)). Writing $\tilde{\alpha}=\sum c_{i} \tilde{\alpha}_{i}$ we can write $\alpha=\sum c_{i} \alpha_{i}$ where all the $c_{i} \in \mathbf{Z}$ and all have the same sign. We call $\alpha=\sum c_{i} \alpha_{i}$ the natural expression of $\alpha$ in terms of $\Pi$. Since $\Pi$ need not consist of linearly independent elements, $\alpha$ can in general be expressed in many ways in terms of the $\left\{\alpha_{i}\right\}$, even with all coefficients of the same sign. However, the natural expression is unique and we will use it when studying the geometry of root data.

We can use Proposition 3 to transfer standard results from $\widetilde{D}$ to $\mathscr{D}$. In particular:

(a) $W$ is a Coxeter group with Coxeter generators $r_{i}, i \in \mathbf{J}$.

(b) Defining for each $w \in W$

$$
S_{w}:=\left\{\alpha \in \Sigma_{+} \mid w^{-1} \alpha \in \Sigma_{-}\right\},
$$


we have $\operatorname{card}\left(S_{w}\right)=l(w)$ where $l$ is the usual length function, and if $w=$ $r_{j_{1}} \cdots r_{j_{n}}$

$$
\begin{gathered}
S_{w}=\left\{\alpha_{j_{1}}, r_{j_{1}} \alpha_{j_{2}}, \ldots, r_{j_{1}} \cdots r_{j_{n-1}} \alpha_{j_{n}}\right\}, \\
S_{w}=\varnothing \Leftrightarrow w=1 .
\end{gathered}
$$

(c) For $i, j \in \mathbf{J}$ and $w \in W$,

$$
w \alpha_{i}= \pm \alpha_{j} \Leftrightarrow w r_{i} w^{-1}=r_{j} .
$$

(d) There is a unique $W$-equivariant bijection

$$
(\cdot)^{\vee}: \Sigma \rightarrow \Sigma^{\vee}
$$

such that

$$
\left(\alpha_{i}\right)^{\vee}=\alpha_{i}^{\vee} \text { for all } i \in \mathbf{J} \text {. }
$$

Furthermore

$$
(\cdot)^{\vee}: \Sigma_{+} \rightarrow \Sigma_{+}^{\vee}
$$

and

$$
\left\langle\alpha,(\alpha)^{\vee}\right\rangle=2 \text { for all } \alpha \in \Sigma \text {. }
$$

In the sequel $(\alpha)^{\vee}$ is denoted simply as $\alpha^{\vee}$. (This notation is consistent with $\left(\alpha_{j}\right)^{\vee}=\alpha_{j}^{\vee}$.) For proofs of these facts one may refer to [MP].

We finish this section with a few words about parabolic subroot data. Let I be a nonempty subset of $\mathbf{J}$ and let

$$
\Pi_{\mathrm{I}}=\left\{\alpha_{i} \mid i \in \mathbf{I}\right\}, \quad \Pi_{\mathrm{I}}^{\vee}=\left\{\alpha_{i}^{\vee} \mid i \in \mathbf{I}\right\} .
$$

If we let $A_{\mathrm{I}}$ be the submatrix of $A$ corresponding to $\mathrm{I}$ it is clear that

$$
D_{\mathbf{I}}=\left(A_{\mathbf{I}}, \Pi_{\mathbf{I}}, \Pi_{\mathrm{I}}^{\vee}, V, V^{\vee},\langle\cdot, \cdot\rangle\right)
$$

satisfies RD1-RD3. We shall see in $\S 6$ that RD4 also holds ${ }^{1}$ and hence that $D_{\mathbf{I}}$ is a set of subroot data of $D$. The Weyl group of $D_{\mathrm{I}}$ is the parabolic subgroup

$$
W_{\mathbf{I}}:=\left\langle r_{i} \mid i \in \mathbf{I}\right\rangle
$$

and its root and coroot systems are given by

$$
\Sigma_{\mathrm{I}}=W_{\mathrm{I}} \Pi_{\mathrm{I}}, \quad \Sigma_{\mathrm{I}}^{\vee}=W_{\mathrm{I}} \Pi_{\mathrm{I}}^{\vee} .
$$

We will let $\Sigma_{\mathbf{I}_{ \pm}}$and $\Sigma_{\mathbf{I}_{ \pm}}^{\vee}$ stand for the obvious objects.

The standard results about parabolic subgroups (see [Bbk] and [Ctr]) also hold in our present situation. For the purpose of this work, however, all we need is the following technical fact.

${ }^{1}$ See $\S 6$, Theorem 1 . That $D_{\mathrm{I}}$ has property RD4 depends only on two technical lemmas about lattices ( $\$ 6$, Lemmas 4 and 5 ). 
Proposition 4. Let $\mathbf{I} \subset \mathbf{J}$ be nonempty. If $\alpha \in \Sigma$ is such that

$$
\alpha=\sum_{i \in \mathbf{I}} c_{i} \alpha_{i}
$$

in natural form (see Corollary to Proposition 3, infra) then $\alpha \in \Sigma_{\mathbf{I}}$.

Proof. We maintain the notation of Proposition 3. By assumption $\tilde{\alpha}=$ $\sum_{i \in \mathbf{I}} c_{i} \tilde{\alpha}_{i} \in \widetilde{\Sigma}$. We assume that $h t(\tilde{\alpha})>0$ and reason by induction on $h t(\tilde{\alpha})$ to conclude that $\tilde{\alpha} \in \widetilde{\Sigma}_{\mathbf{I}}=\widetilde{W}_{\mathbf{I}} \widetilde{\Pi}_{\mathbf{I}}$. Write $\tilde{\alpha}^{\vee}=\sum_{j \in \mathbf{I}} d_{j} \tilde{\alpha}_{j}^{\vee}$ where $d_{j} \geq 0$. Then

$$
2=\left\langle\tilde{\alpha}, \tilde{\alpha}^{\vee}\right\rangle=\sum_{j \in \mathbf{I}} d_{j}\left\langle\tilde{\alpha}, \tilde{\alpha}_{j}^{\vee}\right\rangle
$$

and hence $\left\langle\tilde{\alpha}, \tilde{\alpha}_{i}^{\vee}\right\rangle>0$ for some $i \in \mathbf{I}$. Thus $r_{i} \tilde{\alpha} \in \widetilde{\Sigma}_{\mathbf{I}}$ by induction and hence $\tilde{\alpha} \in \widetilde{\Sigma}_{\mathbf{I}}$. Finally

$$
\alpha=\psi \tilde{\alpha} \in \psi \widetilde{\Sigma}_{\mathbf{I}}=\psi\left(\widetilde{W}_{\mathbf{I}} \widetilde{\Pi}_{\mathbf{I}}\right)=W_{\mathbf{I}} \Pi_{\mathbf{I}}=\Sigma_{\mathbf{I}} .
$$

\section{IMAGINARY ROOTS}

Let $\Sigma$ be the root system of a set of root data $\mathscr{D}=\left(A, \Pi, \Pi^{\vee}, V, V^{\vee}\right.$, $\langle\cdot, \cdot\rangle)$.

If $\varphi \in Q, \alpha \in \Sigma$, and $r_{\alpha} \varphi=\varphi+u \alpha$ for some $u \in \mathbf{Z}$, then the root string between $\varphi$ and $r_{\alpha} \varphi$ is defined as

$$
\left[\varphi, r_{\alpha} \varphi\right]:= \begin{cases}\{\varphi, \varphi+\alpha, \ldots, \varphi+u \alpha\} & \text { if } u \geq 0 \\ \{\varphi, \varphi-\alpha, \ldots, \varphi+u \alpha\} & \text { if } u<0\end{cases}
$$

A subset $\Phi$ of $Q$ is said to have the root string property RSP relative to $\Sigma$ if (RSP1) $\quad \Sigma \subset \Phi$;

(RSP2) whenever $\varphi \in \Phi$ and $\alpha \in \Sigma$ then $\left[\varphi, r_{\alpha} \varphi\right] \subset \Phi$.

There is a unique minimal subset $\Delta$ of $V$ with RSP relative to $\Sigma$. In fact, if we define inductively

$$
\Delta_{0}=\Sigma, \quad \Delta_{n}=\left\{\beta \mid \beta \in\left[\varphi, r_{\alpha} \varphi\right] \text { for some } \alpha \in \Sigma \text { where } \varphi \in \Delta_{n-1}\right\}
$$

then $\Delta_{0} \subset \Delta_{1} \subset \cdots$ and $\Delta:=\bigcup \Delta_{n}$ has the root string property and is minimal.

Henceforth $\Delta$ denotes this minimal set with RSP relative to $\Sigma$. We call $\Delta$ the root string closure of $\Sigma$. We often denote $\Delta \backslash \Sigma$ by ${ }^{\text {im }} \Sigma$.

Proposition 1. $\Delta$ is $W$-invariant.

Proof. We show that $\Delta_{n}$ is $W$-invariant by induction on $n$. This is clear for $\Delta_{0}$. For $\Delta_{n}$, let $\beta \in\left[\varphi, r_{\alpha} \varphi\right]$ where $\varphi \in \Delta_{n-1}, \alpha \in \Sigma$. Let $w \in W$. We have $\beta=\varphi+k \alpha$ where $k \in[0, u]$ and $\left\langle\varphi, \alpha^{\vee}\right\rangle=u$. Then

$$
\left[w \varphi, w r_{\alpha} \varphi\right]=\left[w \varphi, w r_{\alpha} w^{-1} w \varphi\right]=\left[w \varphi, r_{w \alpha} w \varphi\right] \subset \Delta_{n}
$$

(by the induction assumption $\left.w \varphi \in \Delta_{n-1}\right)$. Since $\left[w \varphi, w r_{\alpha} \varphi\right]=[w \varphi, w \varphi+$ $u w \alpha]$, it follows that $w \beta=w \varphi+k w \alpha \in\left[w \varphi, w r_{\alpha} \varphi\right] \subset \Delta_{n}$. 
Proposition 2. If $\Sigma={ }^{\mathrm{re}} \Delta$ where $\Delta$ is the root system of a Kac-Moody Lie algebra then the root string closure of $\Sigma$ is $\Delta$.

Proof. Let $\Phi$ denote the root string closure of $\Sigma$. We already know that $\Delta \supset$ ${ }^{\mathrm{re}} \Delta$ and $\Delta$ has RSP. Thus $\Phi \subset \Delta$. We show that $\Delta_{+} \backslash \Phi=\varnothing$. Similarly $\Delta-\mid \Phi=\varnothing$.

If $\Delta_{+} \backslash \Phi \neq \varnothing$ choose $\beta \in \Delta_{+} \backslash \Phi$ of minimal height. Note that ht $\beta>1$. Then by [Mdy] either

(i) there exists $\alpha \in \Pi$ such that ht $r_{\alpha} \beta<\mathrm{ht} \beta$, in which case $r_{\alpha} \beta \in \Phi$ so also $\beta \in \Phi$ by Proposition 1 or

(ii) for all $\alpha \in \Pi,\left\langle\beta, \alpha^{\vee}\right\rangle \leq 0$ and for at least one $\alpha \in \Pi$ we have $\beta-\alpha \in \Delta_{+}$. In this case $\beta-\alpha \in \Phi$ and since $\left\langle\beta-\alpha, \alpha^{\vee}\right\rangle \leq-2$, $\beta \in\left[\beta-\alpha, r_{\alpha}(\beta-\alpha)\right] \subset \Phi$.

Let $\mathscr{D}$ be a set of root data as above and let $\widetilde{D}$ be its covering. We maintain the notation of $\S 2$, Proposition 3.

Proposition 3. Let $\tilde{\Delta}$ and $\Delta$ be the root string closures of $\widetilde{\Sigma}$ and $\Sigma$ and let $\left\{\tilde{\Delta}_{n}\right\}$ and $\left\{\Delta_{n}\right\}$ be the sets as defined above that define $\tilde{\Delta}$ and $\Delta$ respectively. Then

$$
\psi \tilde{\Delta}_{n}=\Delta_{n} \text { for all } n \in \mathbf{N} \text { and } \psi \widetilde{\Delta}=\Delta .
$$

Proof. $\psi \widetilde{\Delta}_{0}=\psi \widetilde{\Sigma}=\Sigma=\Delta_{0}$. Suppose that $\psi \widetilde{\Delta}_{n-1}=\Delta_{n-1}$.

For $\tilde{\lambda} \in \widetilde{Q}, \lambda \in Q$ with $\psi \tilde{\lambda}=\lambda$ and for $\tilde{\alpha} \in \widetilde{\Sigma}, \alpha \in \Sigma$ with $\psi \tilde{\alpha}=\alpha$ we have

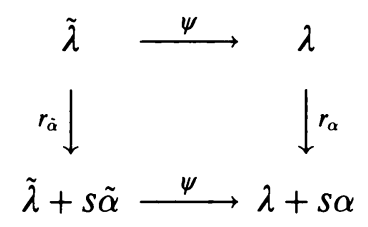

and $\psi\left(\left[\tilde{\lambda}, r_{\tilde{\alpha}} \tilde{\lambda}\right]\right)=\left[\lambda, r_{\alpha} \lambda\right]$.

Thus if $\tilde{\beta} \in \widetilde{\Delta}_{n}, \tilde{\beta} \in\left[\tilde{\lambda}, r_{\tilde{\alpha}} \tilde{\lambda}\right]$ for some $\tilde{\lambda} \in \widetilde{\Delta}_{n-1}, \tilde{\alpha} \in \widetilde{\Sigma}$, and $\beta=\psi(\tilde{\beta}) \in$ $\left[\lambda, r_{\alpha} \lambda\right] \subset \Delta_{n}$. Conversely if $\beta \in \Delta_{n}$ then $\beta \in\left[\lambda, r_{\alpha} \lambda\right]$ for some $\lambda \in \Delta_{n-1}$, $\alpha \in \Sigma$, and choosing preimages $\tilde{\lambda} \in \widetilde{\Delta}_{n-1}, \tilde{\alpha} \in \widetilde{\Sigma}$ we find $\tilde{\beta} \in\left[\tilde{\lambda}, r_{\tilde{\alpha}} \tilde{\lambda}\right]$ with $\psi \tilde{\beta}=\beta$. This shows $\tilde{\psi} \tilde{\Delta}_{n}=\Delta_{n}$ for all $n \in \mathbf{N}$ and hence that $\psi \widetilde{\Delta}=\Delta$.

Remark. The map $\psi: \widetilde{\Delta} \rightarrow \Delta$ need not be injective. For example, consider the Cartan matrix

$$
B^{\prime}:=\left[\begin{array}{rrrr}
2 & -2 & -14 & -34 \\
-2 & 2 & -2 & -14 \\
-14 & -2 & 2 & -2 \\
-34 & -14 & -2 & 2
\end{array}\right]
$$

which, as we saw in Example 1, is of row rank 3. With the notation of Example 1 the set $\Gamma=\left\{\beta_{0}, \beta_{1}, \beta_{2}, \beta_{3}\right\}$ provides us with a set of root data $\mathscr{D}^{\prime}=\left(B^{\prime}, \Gamma, \Gamma^{\vee}, V, V^{\vee},\langle\cdot \mid \cdot\rangle\right)$. Call the resulting root system $\Sigma^{\prime}$ and observe that $\beta_{0}-3 \beta_{1}+3 \beta_{2}-\beta_{3}=0$. 
Let $\widetilde{D}^{\prime}$, with root system $\widetilde{\Sigma}^{\prime}$, be a covering of $\mathscr{D}^{\prime}$ and let $\widetilde{\Delta}^{\prime}$ and $\Delta^{\prime}$ be the root closures of $\widetilde{\Sigma}^{\prime}$ and $\Sigma^{\prime}$. We know that $\widetilde{\Sigma}^{\prime} \stackrel{\psi}{\rightarrow} \Sigma^{\prime}$ is injective. However, $\widetilde{\Delta}^{\prime} \stackrel{\psi}{\rightarrow} \Delta^{\prime}$ is not injective. Indeed $\tilde{\gamma}:=\tilde{\beta}_{1}+\tilde{\beta}_{2}+\tilde{\beta}_{3}+\tilde{\beta}_{4}$ has connected support and satisfies $\left\langle\tilde{\gamma}, \tilde{\beta}_{i}^{\vee}\right\rangle<0, i=1, \ldots, 4$. Thus $\tilde{\gamma} \in \tilde{\Delta}^{\prime}$ by the well-known criterion of $\mathrm{Kac}[\mathrm{Kac}]$. For the same reason $33 \tilde{\gamma} \pm n\left(\tilde{\beta}_{0}-3 \tilde{\beta}_{1}+3 \tilde{\beta}_{2}-\tilde{\beta}_{3}\right) \in \tilde{\Delta}^{\prime}$ for $|n| \leq 10$ and all 21 of these roots have the same image in $\Delta^{\prime}$.

Corollary. $\left.\psi\right|_{\mathrm{im} \widetilde{\Sigma}}:{ }^{\mathrm{im}} \widetilde{\Sigma} \rightarrow{ }^{\mathrm{im}} \Sigma$ and $\psi^{-1}\left({ }^{\mathrm{im}} \Sigma\right) \cap \widetilde{\Delta}={ }^{\mathrm{im}} \widetilde{\Sigma}$.

Proof. Suppose that $\tilde{\gamma} \in{ }^{\operatorname{im}} \widetilde{\Sigma}$ and $\gamma:=\psi(\tilde{\gamma}) \in \Sigma$. Using $W$ we can suppose that $\gamma=\alpha_{i} \in \Pi$. Writing $\tilde{\gamma}=\sum c_{j} \tilde{\alpha}_{j}$, all $c_{j}$ of the same sign, we have $\gamma=\sum c_{j} \alpha_{j}=\alpha_{i}$, which contradicts WIP.

Conversely let $\gamma \in{ }^{\mathrm{im}} \Sigma$. If $\psi(\tilde{\gamma})=\gamma$ and $\tilde{\gamma} \in \widetilde{\Sigma}$ then $\gamma \in \Sigma$, which contradicts ${ }^{\mathrm{im}} \Sigma=\Delta \backslash \Sigma$.

\section{MORPHISMS AND EXTENSION OF THE BASE FIELD}

Throughout this section $\mathscr{D}=\left(A, \Pi, \Pi^{\vee}, V, V^{\vee},\langle\cdot, \cdot\rangle\right)$ and $\mathscr{D}^{\prime}=\left(A^{\prime}, \Pi^{\prime}\right.$, $\left.\Pi^{\prime \vee}, V^{\prime}, V^{\prime \vee},\langle\cdot, \cdot\rangle\right)$ will denote two sets of root data over $\mathbf{K}$. We will use the symbol ' to denote the objects of $\mathscr{D}^{\prime}$. For example,

$$
\Pi^{\prime}=\left\{\alpha_{j^{\prime}}^{\prime}\right\}_{j^{\prime} \in \mathbf{J}^{\prime}} .
$$

By a morphism of $\mathscr{D}$ into $\mathscr{D}^{\prime}$ we will understand a pair $\left(\varphi, \varphi^{\vee}\right)$ of linear maps

$$
\varphi: Q_{\mathbf{K}} \rightarrow Q_{\mathbf{K}}^{\prime} \text { and } \varphi^{\vee}: Q_{\mathbf{K}}^{\vee} \rightarrow Q_{\mathbf{K}}^{\prime \vee}
$$

such that

$\left(\right.$ MOR1) $\quad \varphi(\Pi) \subset \Pi^{\prime}$ and $\varphi^{\vee}\left(\Pi^{\vee}\right) \subset \Pi^{\prime \vee}$,

(MOR2) $\langle x, y\rangle=\left\langle\varphi(x), \varphi^{\vee}(y)\right\rangle^{\prime}$ for all $x \in Q_{\mathbf{K}}$ and $y \subset Q_{\mathbf{K}}^{\vee}$.

Observe that the covering map is a morphism. The concept of isomorphism and automorphism between sets of root data is defined in the obvious fashion. Observe that the ambient spaces $V$ and $V^{\prime}, V^{\vee}$ and $V^{\prime \vee}$ are not required to be isomorphic.

Let $\left(\varphi, \varphi^{\vee}\right)$ be a morphism of $\mathscr{D}$ into $\mathscr{D}^{\prime}$. It is easy to verify that for $\alpha \in \Pi, \alpha^{\prime} \in \Pi^{\prime}$

$$
\varphi(\alpha)=\alpha^{\prime} \Leftrightarrow \varphi\left(\alpha^{\vee}\right)=\alpha^{\prime \vee},
$$

and hence that

$$
\varphi: \Pi \hookrightarrow \Pi^{\prime} \text { and } \varphi^{\vee}: \Pi^{\vee} \hookrightarrow \Pi^{\prime \vee}
$$

are injective maps. In view of this we will henceforth identify $\mathbf{J}$ with a subset of $\mathbf{J}^{\prime}$. 
Lemma 1. Let $\left(\varphi, \varphi^{\vee}\right): \mathscr{D} \rightarrow \mathscr{D}^{\prime}$ be a morphism. Then

(i) $\varphi$ maps $\Sigma$ injectively into $\Sigma^{\prime}$;

(ii) $\varphi(\Delta) \subset \Delta^{\prime}$ where $\Delta$ and $\Delta^{\prime}$ denote the string closures of $\Sigma$ and $\Sigma^{\prime}$ respectively;

(iii) $W$ can be identified with a subgroup of $W^{\prime}$.

Proof. Let $\widetilde{D}$ and $\widetilde{D}^{\prime}$ be the universal coverings of $\mathscr{D}$ and $\mathscr{D}^{\prime}$ respectively. We then have the following commutative diagram:

$$
\begin{aligned}
& \widetilde{\mathscr{D}} \stackrel{\left(\tilde{\varphi}, \tilde{\varphi}^{\vee}\right)}{\longrightarrow} \widetilde{\mathscr{D}}^{\prime} \\
& \left(\psi, \psi^{\vee}\right) \downarrow \quad \downarrow\left(\psi^{\prime}, \psi^{\prime \vee}\right) \\
& \mathscr{D} \stackrel{\left(\varphi, \varphi^{\vee}\right)}{\longrightarrow} \mathscr{D}^{\prime}
\end{aligned}
$$

where $\left(\tilde{\varphi}, \tilde{\varphi}^{\vee}\right)$ is given by the subalgebra relationship between the Kac-Moody Lie algebras of $\mathscr{D}$ and $\mathscr{D}^{\prime}$. The lemma now follows from $\S 2$, Proposition 3 and $\S 3$, Proposition 3 .

Next we consider the set of root data obtained by extending or restricting our base field $\mathbf{K}$. Let $\mathbf{F}$ be a field containing $\mathbf{K}$. Identify $\Pi$ and $\Pi^{\vee}$ with subsets of $V_{\mathbf{F}}:=V \otimes_{\mathbf{K}} \mathbf{F}$ and $V_{\mathbf{F}}^{\vee}:=V^{\vee} \otimes_{\mathbf{K}} \mathbf{F}$ respectively in the obvious way and extend $\langle\cdot, \cdot\rangle$ F-linearly to a (necessarily nondegenerate) pairing

$$
\langle\cdot, \cdot\rangle_{\mathbf{F}}: V_{\mathbf{F}} \times V_{\mathbf{F}}^{\vee} \rightarrow \mathbf{F} .
$$

Then

$$
\mathscr{D}^{\mathbf{F}}:=\left(A, \Pi, \Pi^{\vee}, V_{\mathbf{F}}, V_{\mathbf{F}}^{\vee},\langle\cdot, \cdot\rangle_{\mathbf{F}}\right)
$$

is clearly a set of root data over $\mathbf{F}$.

Here is how we restrict a set of root data to the field of rational numbers Q. Recall that by RD4 there exist $\left\{\gamma_{i}\right\}_{i \in \mathbf{I}} \subset Q$ and $\left\{\gamma_{i}^{\vee}\right\}_{v} \subset Q^{\vee}$ each linearly independent over $\mathbf{K}$ such that

$$
Q=\bigoplus_{i \in \mathbf{I}} \mathbf{Z} \gamma_{i} \text { and } Q^{\vee}=\bigoplus_{i \in \mathbf{I}} \mathbf{Z} \gamma_{i}^{\vee}
$$

It follows that $\left\langle\gamma_{i}, \gamma_{j}^{\vee}\right\rangle \in \mathbf{Q}$ for all $i, j \in \mathbf{I}$. It is not hard to find a set $\mathbf{M} \supset \mathbf{I}$ and rational spaces

$$
U_{\mathbf{Q}}:=\bigoplus_{j \in \mathbf{M}} \mathbf{Q} \beta_{j}, \quad U_{\mathbf{Q}}^{\vee}:=\bigoplus_{j \in \mathbf{M}} \mathbf{Q} \beta_{j}^{\vee}
$$

and a nondegenerate pairing

$$
\langle\cdot, \cdot\rangle_{\mathbf{Q}}: U_{\mathbf{Q}} \times U_{\mathbf{Q}}^{\vee} \rightarrow \mathbf{Q}
$$

such that

$$
\left\langle\beta_{i}, \beta_{j}^{\vee}\right\rangle_{\mathbf{Q}}=\left\langle\gamma_{i}, \gamma_{j}^{\vee}\right\rangle
$$


for all $i, j \in \mathbf{I}$. (For example, set $\mathbf{K}=\mathbf{I} \cup \mathbf{I}^{\prime}$ where $\mathbf{I}^{\prime}:=\left\{i^{\prime} \mid i \in \mathbf{I}\right\}$. Let $\left\{\beta_{k}\right\}_{k \in \mathbf{K}}$ and $\left\{\beta_{k}^{\vee}\right\}_{k \in \mathbf{K}}$ be the chosen bases of the Q-spaces $U_{\mathbf{Q}}$ and $U_{\mathbf{Q}}^{\vee}$ respectively and define $\langle\cdot, \cdot\rangle_{\mathbf{Q}}: U_{\mathbf{Q}} \times U_{\mathbf{Q}}^{\vee} \rightarrow \mathbf{Q}$ by setting for all $i, j \in \mathbf{I}$ and $i^{\prime}, j^{\prime} \in \mathbf{I}^{\prime}$

$$
\begin{aligned}
\left\langle\beta_{i}, \beta_{j}^{\vee}\right\rangle_{\mathbf{Q}} & =\left\langle\gamma_{i}, \gamma_{j}^{\vee}\right\rangle, \\
\left\langle\beta_{i}, \beta_{j^{\prime}}^{\vee}\right\rangle_{\mathbf{Q}} & =\delta_{i j}=\left\langle\beta_{i^{\prime}}, \beta_{j}^{\vee}\right\rangle_{\mathbf{Q}}, \\
\left\langle\beta_{i^{\prime}}, \beta_{j^{\prime}}^{\vee}\right\rangle_{\mathbf{Q}} & =0 .
\end{aligned}
$$

Then $\langle\cdot, \cdot\rangle_{\mathbf{Q}}$ is as desired.) Let

$$
Q_{\mathbf{Q}}:=\bigoplus_{i \in \mathbf{I}} \mathbf{Z} \beta_{i} \text { and } Q_{\mathbf{Q}}^{\vee}=\bigoplus_{i \in \mathbf{I}} \mathbf{Z} \beta_{i}^{\vee}
$$

We identify $\Pi$ and $\Pi^{\vee}$ with subsets of $Q_{\mathbf{Q}}$ and $Q_{\mathbf{Q}}^{\vee}$ respectively in the obvious way: if $\alpha_{i}=\sum_{\vee} c_{i j} \gamma_{i}$ in $Q$ then $\alpha_{i}$ is identified with $\sum c_{i j} \beta_{j}$ in $Q_{\mathbf{Q}}$, and similarly for $\alpha_{i}^{\vee}$. Clearly

$$
\left\langle\alpha_{i}, \alpha_{j}^{\vee}\right\rangle_{\mathbf{Q}}=A_{i j}
$$

for all $i, j \in \mathbf{J}$. It follows that

$$
\mathscr{D}_{\mathbf{Q}}=\left(A, \Pi, \Pi^{\vee}, U_{\mathbf{Q}}, U_{\mathbf{Q}}^{\vee},\langle\cdot, \cdot\rangle_{\mathbf{Q}}\right)
$$

is a rational set of root data with root system $\Sigma$. Note that $Q=\sum_{\alpha \in \Sigma} \mathbf{Z} \alpha=Q_{\mathbf{Q}}$ so that $Q$ can be identified with $Q_{\mathrm{Q}}$. Similarly $Q^{\vee}$ can be identified with $Q_{\mathrm{Q}}^{\vee}$. Note that $U_{\mathbf{Q}}$ and $U_{\mathbf{Q}}^{\vee}$ are not required to be subsets of $V$ and $V^{\vee}$.

Remark. The following observations will be used in $\S 6$. Let $\mathscr{D}$ be a set of root data over $\mathbf{K}$ as above. For convenience we will refer to the above constructions as field manipulations of $D$. We note that

1. After obvious identification the root and coroot lattice together with their roots and coroots (and hence also $\Pi$ and $\Pi^{\vee}$ ) remain unchanged under field manipulation.

2. The geometric triple $\left(Q, Q^{\vee},\langle\cdot, \cdot\rangle\right)$ remains unchanged under field manipulations.

We admit that this terminology is loose but its meaning is nonetheless precise. From these considerations it is clear that

$$
\mathscr{D}_{\mathbf{Q}}{ }^{\mathbf{K}} \simeq \mathscr{D} \text { and } \mathscr{D}_{\mathbf{Q}}{ }^{\mathbf{K}} \simeq \mathscr{D}_{\mathbf{Q}} \text {. }
$$

Definition. If $\mathscr{D}=\left(A, \Pi, \Pi^{\vee}, V, V^{\vee},\langle\cdot, \cdot\rangle\right)$ is as above, by a set of subroot data of $\mathscr{D}$ we will understand a set of root data of the form

$$
\mathscr{D}^{\prime}=\left(B, \Upsilon, \Upsilon^{\vee}, V, V^{\vee},\langle\cdot, \cdot\rangle\right)
$$

where $B$ is a certain matrix, $\Upsilon \subset \Sigma$, and $\Upsilon^{\vee} \subset \Sigma^{\vee}$. An example of this is given by the Maxwell Demon. Important for us in $\S 6$ is the fact that subroot data transfer via field manipulations. More precisely, we will make use of the 
following fact. If $\mathscr{D}^{\prime}$ is a set of subroot data of the real set of subroot data $D_{\mathbf{Q}}{ }^{\mathbf{R}}$ then $\mathscr{D}^{\prime}$ corresponds to a set of subroot data of $\mathscr{D}_{\mathbf{Q}}{ }^{\mathbf{R}} \mathbf{Q}{ }^{\mathbf{K}} \simeq \mathscr{D}$.

Remark. We can define an abstract root system as follows: A subset $\Sigma$ of a $\mathbf{K}$-space $V$ is called a root system if $\Sigma$ is the set of real roots of a set of root data of the form $\left(A, \Pi, \Pi^{\vee}, V, V^{\vee},\langle\cdot, \cdot\rangle\right)$.

\section{THE GEOMETRY OF A SET OF ROOT DATA}

Let $\mathscr{D}=\left(A, \Pi, \Pi^{\vee}, V, V^{\vee},\langle\cdot, \cdot\rangle\right)$ be a set of root data over $\mathbf{R}$. We maintain all the notation of the previous sections. Throughout the present section we will assume that $\operatorname{dim}_{\mathbf{R}}(V)=n$ is finite. Because of RD2 and RD4 we have

(a) $\operatorname{dim}_{\mathbf{R}}\left(V^{\vee}\right)=n$;

(b) $\mathbf{J}$ is at most countable (though not necessarily finite);

(c) $Q=\bigoplus_{i=1}^{l} \mathbf{Z} \gamma_{i}$ and $Q^{\vee}=\bigoplus_{i=1}^{l} \mathbf{Z} \gamma_{i}^{\vee}$ for some $\left\{\gamma_{i}\right\} \subset Q$ and $\left\{\gamma_{i}^{\vee}\right\} \subset Q^{\vee}$ linearly independent over $\mathbf{R}$.

Henceforth we will think of both $V$ and $V^{\vee}$ as topological vector spaces by identification with $\mathbf{R}^{n}$. If $S \subset \mathbf{R}^{n}$ then $\stackrel{\circ}{S}$ and $\bar{S}$ will denote the interior and closure of $S$ respectively.

Let $h \in V^{\vee}, h \neq 0$. Then $H:=\{x \in V \mid\langle x, h\rangle=0\}$ is a hyperplane of $V$. $H$ decomposes the set $V \backslash H$ into two open half-spaces

$$
H^{ \pm}=\left\{x \in V \mid\langle x, h\rangle \in \pm \mathbf{R}_{>0}\right\} .
$$

Two points $x$ and $y$ of $V \backslash H$ are said to be on the same (respectively opposite) sides of $H$ if $x$ and $y$ lie (respectively do not lie) in one of these two open half-spaces. If $x$ and $y$ in $V \backslash H$ lie on opposite sides of $H$ we say that $H$ separates $x$ and $y$.

Given $\alpha^{\vee} \in \Sigma_{+}^{\vee}$ define $H_{\alpha^{\vee}}:=\left\{x \in V \mid\left\langle x, \alpha^{\vee}\right\rangle=0\right\}$. Let $\mathbf{H}=\left\{H_{\alpha^{\vee}} \mid \alpha \in\right.$ $\left.\Sigma_{+}\right\}$. Following [Bbk], define an equivalence relation $\sim_{\mathbf{H}}$ on $V$ by:

For all $x$ and $y$ in $V$ set $x \sim_{\mathbf{H}} y$ if and only if for all $H \in \mathbf{H}$ either

(i) $x$ and $y$ belong to $H$ or

(ii) $x$ and $y$ lie on the same side of $H$ (in particular, neither $x$ nor $y$ lies in $H$ ).

Clearly $w H_{\alpha^{\vee}}=H_{w \alpha^{\vee}}=H_{-w \alpha^{\vee}}$ and hence $W$ acts on $\mathbf{H}$. Thus $W$ acts on the quotient set $V / \sim$.

The fundamental chamber $F$ of $\mathscr{D}$ is defined by

$$
\begin{aligned}
F & :=\left\{x \in V \mid\left\langle x, \alpha_{j}^{\vee}\right\rangle>0 \text { for all } j \in \mathbf{J}\right\} \\
& =\left\{x \in V \mid\left\langle x, \alpha^{\vee}\right\rangle>0 \text { for all } \alpha \in \Sigma_{+}\right\} .
\end{aligned}
$$

For each subset $\mathbf{I} \subset \mathbf{J}$ define a set $F_{\mathbf{I}}$ by

$$
F_{\mathbf{I}}:=\left\{x \in V \mid\left\langle x, \alpha_{j}^{\vee}\right\rangle>0 \text { if } j \notin \mathbf{I} \text { and }\left\langle x, \alpha_{j}^{\vee}\right\rangle=0 \text { if } j \in \mathbf{I}\right\} \text {. }
$$

Note that $F=F_{\varnothing}$ and that each of the $F_{1}$ is a convex cone.

Since the elements of $\Pi^{\vee}$ are not necessarily linearly independent, there is no guarantee that $F_{\mathbf{I}} \neq \varnothing$ for all $\mathbf{I} \subset \mathbf{J}$. However, 


\section{Proposition 1.}

(i) $\stackrel{\circ}{F} \neq \varnothing$. Moreover $\stackrel{\bar{\circ}}{F}=\bar{F}$.

(ii) For all $k \in \mathbf{J}$ we have that $F_{\{k\}}$ supports the hyperplane $H_{\alpha_{k}^{v}}$.

(iii) $\bar{F}=\bigcup_{\mathbf{I} \subset \mathbf{J}} F_{\mathbf{I}}$.

Proof. (i) Let $\gamma_{1}^{\vee}, \ldots, \gamma_{l}^{\vee}$ be as in RD4. The set $\Lambda=\left\{x \in V \mid\left\langle x, \gamma_{i}^{\vee}\right\rangle>0\right.$, $1 \leq i \leq l\}$ is open and nonempty. Since $\Sigma_{+}^{\vee} \subset \bigoplus_{i=1}^{l} \mathbf{N} \gamma_{i}^{\vee}$ it is clear that $\Lambda \subset F$. Thus $F^{0} \neq \varnothing$. We claim that for all $0 \leq t<1$

$$
t \bar{F}+(1-t) F^{0} \subset F^{0} \text {. }
$$

Since the left-hand side is open it will suffice to show that it lies in $F$. Now if $\gamma \in \bar{F}$ then $\left\langle x, \alpha_{j}^{\vee}\right\rangle \geq 0$ for all $j \in \mathbf{J}$. Let $y \in F^{0}$ and $0 \leq t<1$. Then $\left\langle t x+(1-t) y, \alpha_{j}^{\vee}\right\rangle>0$ for all $j \in \mathbf{J}$. This establishes our claim. Finally, if $x \in \bar{F}$ and $y \in F^{0}$ then $\left.] x, y\right] \subset F^{0}$ so that $x \in \overline{F^{0}}$.

(ii) Let $B$ be an open ball in $F$. For each $x \in B, r_{k} x$ satisfies the inequalities

$$
\left\langle r_{k} x, \alpha_{j}^{\vee}\right\rangle>0, \quad j \neq k .
$$

The same goes for every point of the interior $U$ of the convex hull of $B \cup r_{k} B$. Now $U \cap H_{\alpha_{k}^{\vee}}$ is a nonempty subset of $F_{\{k\}}$, from which (ii) follows.

(iii) Let $x \in F_{\mathrm{I}}$. If $y \in F$ then $] x, y[\subset F$. Thus $x \in \overline{x, y[} \subset \bar{F}$. This shows that $\bigcup_{\mathbf{I} \subset \mathbf{J}} F_{\mathbf{I}} \subset \bar{F}$. The reverse inclusion is clear.

Proposition 2. For each subset $\mathbf{I}$ of $\mathbf{J}$ for which $F_{\mathbf{I}} \neq \varnothing, F_{\mathbf{I}}$ is an equivalence class of $\sim_{\mathbf{H}}$.

Proof. Let $x \in F_{\mathrm{I}}$ and let $\tilde{x}$ denote the equivalence class of $x$ in $V$. Evidently $\tilde{x} \subset F_{\mathrm{I}}$ since $F_{\mathrm{I}}$ is defined by a subset of the relations defining $\tilde{x}$. Now suppose that $y \in F_{\mathbf{I}}$ and let $\alpha^{\vee} \in \Sigma^{\vee}$. Since $H_{\alpha^{\vee}}=H_{-\alpha^{\vee}}$ we can suppose that $\alpha^{\vee} \in \Sigma_{+}^{\vee}$ and write $\alpha^{\vee}=\sum_{j \in \mathrm{J}} c_{j} \alpha_{j}^{\vee}, c_{j} \geq 0$. Then $\left\langle x, \alpha^{\vee}\right\rangle=\sum_{j \in \mathrm{J}} c_{j}\left\langle x, \alpha_{j}^{\vee}\right\rangle \geq 0$ with equality if and only if $j \in \mathbf{I}$ whenever $c_{j} \neq 0$. It follows that $\left\langle y, \alpha^{\vee}\right\rangle \geq 0$, and $\left\langle y, \alpha^{\vee}\right\rangle=0 \Leftrightarrow\left\langle x, \alpha^{\vee}\right\rangle=0$. Thus either $x$ and $y$ both lie in $H_{\alpha^{\vee}}$ or both lie on the same side of $H_{\alpha^{v}}$. This proves $y \sim x$.

Proposition 3. Let $\mathbf{I}, \mathbf{I}^{\prime} \subset \mathbf{J}$ and let $w \in W$. If $w F_{\mathbf{I}} \cap F_{\mathbf{I}^{\prime}} \neq \varnothing$ then $\mathbf{I}=\mathbf{I}^{\prime}$, $w F_{\mathbf{I}}=F_{\mathbf{I}^{\prime}}=F_{\mathbf{I}}$, and $w \in W_{\mathbf{I}}$. Moreover $w$ fixes $F_{\mathbf{I}}$ pointwise.

Proof. This is standard [Bbk]. The key observation is that $l\left(r_{i} w\right)<l(w) \Rightarrow$ $w F_{\mathbf{I}} \subset H_{\alpha_{i}}^{-}$.

We introduce the standard terminology as found in [Bbk]. Since $W$ permutes the set of hyperplanes $\mathbf{H}, W$ preserves the equivalence relation $\sim_{\mathbf{H}}$ and hence permutes the equivalence classes of $\sim_{\mathbf{H}}$. In particular, each of the sets $w F_{\mathbf{I}}$, $\mathbf{I} \subset \mathbf{J}$, is an equivalence class if it is not empty. We call these particular 
equivalence classes facettes. The union of all the facettes is the Tits cone

$$
\begin{aligned}
\mathscr{X} & :=\bigcup\left\{w F_{\mathbf{I}} \mid w \in W, \mathbf{I} \subset \mathbf{J}\right\} \\
& =\bigcup\{w \bar{F} \mid w \in W\} .
\end{aligned}
$$

Since each $F_{\mathbf{I}}$ is a cone it is evident that $\mathscr{X}$ is indeed a cone. Furthermore, $\mathscr{X}$ is $W$-invariant. Since $F$ contains an open subset of $V$, the interior $\mathscr{\mathscr { X }}$ of $\mathscr{Z}$ is nonempty. If two facettes $w F_{\mathrm{I}}$ and $w^{\prime} F_{\mathbf{I}^{\prime}}$ intersect nontrivially, then, by Proposition 3, $\mathbf{I}=\mathbf{I}^{\prime}$. Thus each facette has a well-defined subset $\mathbf{I}$ of $\mathbf{J}$ associated with it called the type of the facette. The facettes of type $\varnothing$, which are of the form $w F_{\varnothing}=w F$, are called the chambers of $\mathscr{X}$. These are precisely the facettes that contain open subsets of $V$. The facettes of type $\{i\}, i \in \mathbf{I}$, are called faces of $\mathscr{X}$.

From Proposition 1(ii) it follows that every face supports a hyperplane and it is elementary that faces are the only facettes supporting hyperplanes.

The faces $F_{\{i\}}, i \in \mathbf{J}$, all lie in $\bar{F}$. Indeed these are the only faces lying in $\bar{F}$, for if $x \in \bar{F}$ then $x \in F_{\mathbf{I}}$ for some I. If also $x \in w F_{\{j\}}$ for some $w \in W$ and $j \in \mathbf{J}$ then by Proposition $3,\{j\}=\mathbf{I}$ and $w F_{\{j\}}=F_{\{j\}}$. The faces lying in $\overline{w F}$ for any $w$ are then $\left\{w F_{\{i\}} \mid i \in \mathbf{I}\right\}$. These are called the faces of the chamber $w F$. The hyperplanes $w H_{\alpha_{i}^{\vee}}$ supported by these faces are all called the walls of the chamber.

Example 5. We begin by showing that the chambers of the Demon are not open. We maintain the notation of Examples 1 and 3. For a point $a \alpha_{0}+b \alpha_{1}+c \alpha_{2}$ to belong to $F$ we must have

$$
\left\langle a \alpha_{0}+b \alpha_{1}+c \alpha_{2}, \beta_{k}^{\vee}\right\rangle>0 \text { for all } k \in \mathbf{Z} .
$$

Using the expressions for $\beta_{k}^{\vee}$ this translates into

$$
-2 a k^{2}+2(c-b) k+(a-b)>0 \text { for all } k \in \mathbf{Z} .
$$

Thus $-\alpha_{1}-\alpha_{2} \in F$ but it is clear that no neighborhood of $-\alpha_{1}-\alpha_{2}$ can be contained in $F$.

What follows is a description of the chamber geometry of the Demon in the Poincare model of the upper half-plane.

The interior of the Tits cone $\mathscr{X}$ for the root system defined by

$$
A=\left[\begin{array}{rrr}
2 & -2 & 0 \\
-2 & 2 & -2 \\
0 & -2 & 2
\end{array}\right]
$$

is the set of points $x=\left(x_{0}, x_{1}, x_{2}\right)$ satisfying

$$
x_{0}^{2}-2 x_{0} x_{1}+x_{1}^{2}-2 x_{1} x_{2}+x_{2}^{2}<0, \quad x_{1}<0,
$$

with fundamental region $F$ bounded by the rays through $(0,-1,-1),(-1$, $-1,-1),(-1,-1,0)$. Projectivizing and setting $X_{i}=\left(x_{i} / x_{1}\right)-1, i=0,2$, we 
have $\mathscr{X} / \mathbf{R}^{\times}$given as the interior of the disk $X_{0}^{2}+X_{2}^{2}<1$. Identifying this with the disk $D=\{z|| z \mid<1\} \subset \mathbf{C}$, the three rays determine the points $-1,0,-i$ whose convex hull is the image of $F$. One passes from the Klein model to the Poincaré model by the transformation $z \mapsto 2 z /(1+z \bar{z})$, which fixes the boundary of $D$ pointwise. Following this by the Möbius transformation $z \mapsto$ $i(z+1) /(-z+1)$ we arrive at the upper half-plane model of the hyperbolic plane $\mathscr{H}$. The fundamental region is now the triangle with vertices $0, i, 1$ respectively. The reflections $r_{i}, i=1,2$, appear here as inversions in the circles whose diameters are 0,1 and $0, \infty$ respectively, namely

$$
r_{1}: z \mapsto \bar{z} / 2 \bar{z}-1, \quad r_{2}: z \mapsto-\bar{z}, \quad r_{2} r_{1}: z \rightarrow z / 1-2 z
$$

The walls of the fundamental chamber $C$ of the Demon are given by the hyperplanes $H_{\alpha}, \alpha=\left(r_{2} r_{1}\right)^{k} \alpha_{0}, k \in \mathbf{Z}$. In $\mathscr{H}, H_{\alpha_{0}}$ appears as the orthogonal semicircle $\gamma$ with diameter $[-1,1]$. The translates of this by $\left(r_{2} r_{1}\right)^{k}$ yield the semicircles on diameters $[1 /(2 n+1), 1 /(2 n-1)], n \in \mathbf{Z}$. This realizes the fundamental region of the Demon as the connected region $\widetilde{C}$ in white beneath the semicircle $\gamma$ (see Figure 1). Inversion in $\gamma$ yields the fundamental region as the extended black region $\widetilde{C}^{\prime}$ in which its infinite symmetry is clearly revealed. One observes that $O$ is not separated from $\widetilde{C}$ by any wall of $\widetilde{C}$, nor is it on a wall of $\widetilde{C}$. Thus the ray $\mathbf{R}_{+}(0,-1,-1)$ lies in the fundamental region of the Demon (in $\mathbf{R}^{3}$ ) while the ray $\mathbf{R}_{+}(-1,-1,0)$ lies only in its closure. The Tits cone for the Demon is, as a point set, exactly the set $\mathscr{X}$.

The geometry of the Demon we have described is related to the Schwarz triangle with vertices in $-1,1, \infty$ and sum of its interior angles equal to zero [Ctd, p. 184].

For each $\mathbf{I} \subset \mathbf{J}$ let $W_{\mathbf{I}}$ be the parabolic subgroup

$$
W_{\mathbf{I}}:=\left\langle r_{i} \mid i \in \mathbf{I}\right\rangle \text {. }
$$

Proposition 4. Let the notation be as above. Then

(i) $\mathscr{X}=\left\{x \in V \mid\left\langle x, \alpha^{\vee}\right\rangle<0\right.$ for a finite number of $\left.\alpha \in \Sigma_{+}\right\}$.

(ii) $\mathscr{X}$ is a convex cone. A similar result applies to $\mathscr{X}^{\vee}$.

Proof. The proof is the usual one (see [Kac, MP]).

Proposition 5. Let $x, y \in \mathscr{X}$. The following are equivalent.

(i) There exists $w \in W$ such that both $x$ and $y$ belong to $w \bar{F}$.

(ii) There exists no hyperplane of $\mathbf{H}$ separating $x$ and $y$.

Proof.

(i) $\Rightarrow$ (ii) Clear.

(ii) $\Rightarrow$ (i) Consider the open interval $] x, y$ [. We claim that if $p, q \in] x, y[$ then $p \sim q$. For if not we may assume that there exists $H \in \mathbf{H}$ such that either $p \in H^{+}$and $q \in H^{-}$or $p \in H$ and $q \in H^{-}$, and it follows in either case that 


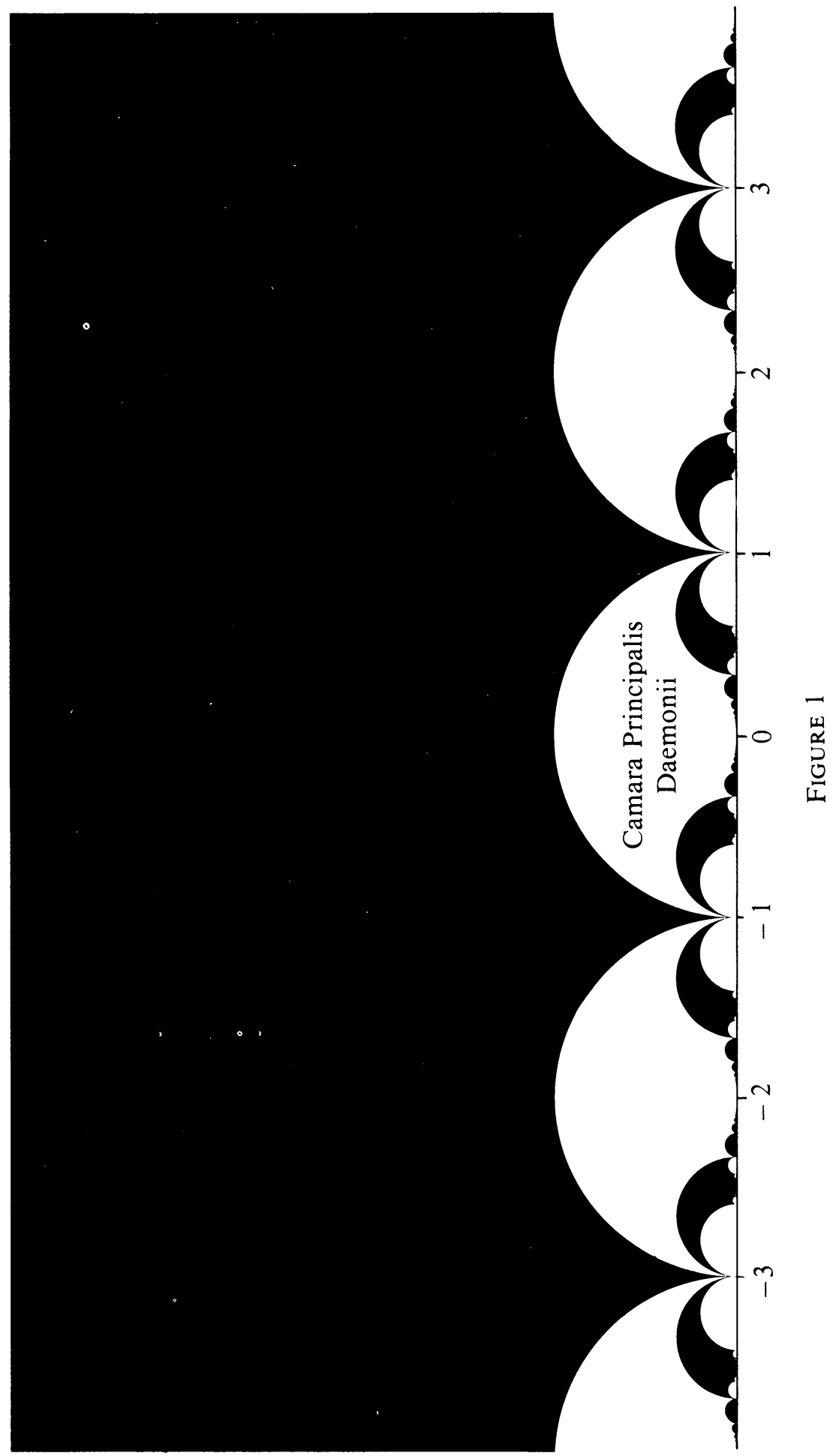


$H$ separates $x$ and $y$. Since $] x, y[\subset \mathscr{X}$ it follows that $] x, y[$ is included in one equivalence class of $\mathscr{X}$. Thus

$$
] x, y\left[\subset w F_{\mathbf{I}}\right.
$$

for some $w \in W$ and $\mathbf{I} \subset \mathbf{J}$. Hence

$$
[x, y] \subset \overline{w F}_{\mathbf{I}}=w \bar{F}_{\mathbf{I}} \subset w \bar{F} .
$$

Proposition 6. Let $x, y \in \mathscr{X}$ and $w \in W$.

(i) There exists a finite number \# $(x, y)$ of hyperplanes of $\mathbf{H}$ separating $x$ and $y$.

(ii) If $x \in F$ and $y=w x$ then $\#(x, y)=l(w)$.

Proof. Recall that the elements of $\mathbf{H}$ are in one-to-one correspondence with the elements of $\Sigma_{+}$. Since $W$ stabilizes both $\mathscr{X}$ and $\mathbf{H}$ we can assume that $x \in \bar{F}$ and $y=w z$ for some $z \in \bar{F}$. Let $\alpha^{\vee} \in \Sigma_{+}$. Then

$$
\begin{aligned}
& H_{\alpha^{\vee}} \text { separates } x \text { and } y \Leftrightarrow \\
& \left\langle x, \alpha^{\vee}\right\rangle>0 \text { and }\left\langle y, \alpha^{\vee}\right\rangle<0 \Leftrightarrow \\
& \left\langle x, \alpha^{\vee}\right\rangle>0 \text { and }\left\langle z, w^{-1} \alpha^{\vee}\right\rangle<0 \Leftrightarrow \\
& \left\langle x, \alpha^{\vee}\right\rangle>0 \text { and } w^{-1} \alpha^{\vee} \in \Sigma_{-}^{\vee} \Leftrightarrow \\
& \left\langle x, \alpha^{\vee}\right\rangle>0 \text { and } w^{-1} \alpha \in \Sigma_{-} \Leftrightarrow \\
& \left\langle x, \alpha^{\vee}\right\rangle>0 \text { and } \alpha \in S_{w} .
\end{aligned}
$$

Both (i) and (ii) now follow from the fact that Card $S_{w}=l(w)$. (See $\S 2$.)

Proposition 7. Let $x \in \stackrel{\circ}{\mathscr{X}}$. The set

$$
\mathbf{H}_{x}:=\{H \in \mathbf{H} \mid x \in H\}
$$

is finite.

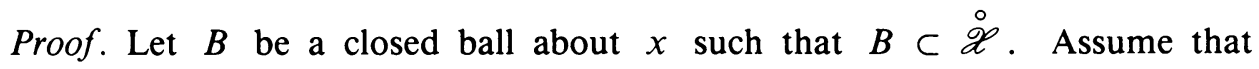
$\left\{H_{i}\right\}_{i=1, \infty} \subset \mathbf{H}$ is a sequence of distinct hyperplanes such that $x \in H_{i}$ for all $i>0$.

Let $S_{i}:=H_{i} \backslash\left(\bigcup_{j \neq i}\left(H_{i} \cap H_{j}\right)\right)$. By Baire category each $S_{i}$ is infinite. Fix $i$ and let $y \in S_{i}$. We claim that $L(y):=\{(1-\lambda) x+\lambda y \mid \lambda \in \mathbf{R} \backslash\{0\}\} \subset S_{i}$ (i.e., the line through $x$ and $y$ lies entirely in $\left.S_{i} \cup\{x\}\right)$. Indeed $L(y) \subset H_{i}$, so if $L(y) \not \subset S_{i}$ there exist $\lambda \neq 0$ and $j \neq i$ such that $(1-\lambda) x+\lambda y \in H_{j}$. But $(1-\lambda) x \in H_{j}$. Thus $\lambda y \in H_{j}$ and hence $y \in H_{j}$. This contradicts the assumption $y \in S_{i}$ and establishes our claim.

The line $L(y)$ intersects the frontier of $B$ at two (diametrically opposed) points that we will denote by $c_{i}$ and $d_{i}$. We have shown that for all $i \neq j$

$$
c_{i}, d_{i} \in H_{i} \text { and } c_{i}, d_{i} \notin H_{j} .
$$


However, it is clear that if $j \neq 1$ then $H_{j}$ separates $c_{1}$ and $d_{1}$. This contradicts Proposition 6 and hence establishes our result.

Proporition 8. Let $\mathscr{Z}$ be the Tits cone and let $F$ be its fundamental chamber.

(i) For all $x, y \in \mathscr{X}$ the closed interval $[x, y]$ lies inside the union of finitely many facettes.

(ii) If $w \in W$ and $\mathbf{I} \subset \mathbf{J}$ are such that $w F_{\mathbf{I}} \cap \bar{F} \neq \varnothing$ then $w \in W_{\mathbf{I}}$ and $w$ fixes $F_{\mathrm{I}}$ pointwise.

(iii) If $w \in W$ fixes $x \in \mathscr{X}$ then $w$ fixes pointwise the facette containing $x$.

(iv) The following conditions hold.

(FD1) $W$ acts simply transitively on the set of chambers.

(FD2) If $x \in \mathscr{Z}$ there exists a unique $z \in \bar{F}$ such that $x \in W z$.

In particular, $F$ is a fundamental region for the action of $W$ on $\mathscr{X}$.

Proof. The only part that needs some explanation is (i). Let $x, y \in \mathscr{X}, x \neq y$. The open interval $] x, y[$ is cut by precisely those hyperplanes separating $x$ and $y$, of which there is only a finite number. Each cut point lies in a single facette and so also does each open interval between two cut points. Finally, $x$ and $y$ each lie in a single facette.

Remark. At this point we have established the axioms of [MY] (notably Proposition 11(i), (ii)).

If $Y$ is a nonempty subset of $V$ we define

$$
\begin{aligned}
& \mathbf{H}_{Y}:=\{H \in \mathbf{H} \mid Y \subset H\}, \\
& W^{Y}:=\{w \in W \mid w y=y \text { for all } y \in Y\}, \\
& {[Y]:=\text { the subspace of } V \text { spanned by } Y .}
\end{aligned}
$$

Proposition 9. Let $Y$ be a nonempty subset of $\mathscr{X}$ and let $K=\bigcap_{H \in \mathrm{H}_{Y}} H$. There exist $w \in W$ and $\mathbf{I} \subset \mathbf{J}$ such that

(i) $W^{w F_{1}}=W^{Y}=W^{K}=w W_{1} w^{-1}$;

(ii) $\mathbf{H}_{Y}=w\left\{H_{\alpha^{\vee}} \mid \alpha^{\vee} \in \Sigma_{\mathbf{I}}^{\vee}\right\}$.

In particular, the following three groups are equal:

1. the elements of $W$ that fix $Y$ pointwise;

2. the subgroup of $W$ generated by all reflections $r_{\alpha}$ such that $r_{\alpha}$ fixes $Y$ pointwise;

3. the subgroup of $W$ generated by all reflections $r_{\alpha}$ such that $Y \subset H_{\alpha^{v}}$. Proof. Choose $x \in[Y] \cap \mathscr{X}$ such that $x \notin H$ for all $H \in \mathbf{H} \backslash \mathbf{H}_{Y}$. By means of $W$ we can assume that $x \in F_{\mathrm{I}}$ for some $\mathbf{I} \subset \mathbf{J}$. By Proposition 8(ii)

$$
W^{\{x\}}=W_{\mathbf{I}}=W^{F_{1}} .
$$

Let $\alpha^{\vee} \in \Sigma_{+}^{\vee}$ be such that $H_{\alpha^{\vee}} \in \mathbf{H}_{Y}$. Then $x \in[Y] \Leftrightarrow\left\langle x, \alpha^{\vee}\right\rangle=0$. Write $\alpha^{\vee}=\sum c_{j} \alpha_{j}^{\vee}, c_{j} \in \mathbf{N}$ in natural form (see $\left.\S 2\right)$. Using the definition of $F_{\mathbf{I}}$ we 
find that $c_{j}=0$ if $j \notin I$. Thus $\alpha^{\vee} \in \Sigma_{\mathbf{I}}^{\vee}(\S 2$, Proposition 4). On the other hand

$$
\alpha^{\vee} \in \Sigma_{\mathbf{I}}^{\vee} \Leftrightarrow\left\langle x, \alpha^{\vee}\right\rangle=0 \Rightarrow x \in H_{\alpha^{\vee}} \Rightarrow H_{\alpha^{\vee}} \in \mathbf{H}_{Y} .
$$

Thus $\mathbf{H}_{Y}=\left\{H_{\alpha^{\vee}} \mid \alpha^{\vee} \in \Sigma_{\mathbf{I}}\right\}$. It follows that for all $i \in \mathbf{I}, r_{\alpha_{i}}$ fixes $K$ pointwise and hence that $W^{F_{1}} \subset W^{K}$. The rest of the proof is standard.

Corollary. If $r \in W$ is a reflection then $r=r_{\alpha}$ for some $\alpha \in \Sigma$.

Proof. Let $H$ be the hyperplane of fixed points of $r$. It is trivial to see that $H \cap \stackrel{\circ}{\mathscr{X}} \neq \varnothing$ and hence $H \cap \stackrel{\circ}{\mathscr{X}}$ spans $H$. By the proposition, $W^{H}$ is generated by the reflections $r_{\alpha}$ for which $H=H_{\alpha^{\vee}}$. It follows at once that $\alpha$ is unique, up to sign, and hence that $w=r_{\alpha}$.

Proposition 10. Let $x \in \stackrel{\circ}{\mathscr{X}}$.

(i) There exists an open convex set $B$ in $\stackrel{\circ}{\mathscr{X}}$ containing $x$ and satisfying

$$
H \cap B \neq \varnothing \Leftrightarrow x \in H \quad \text { for all } H \in \mathbf{H} .
$$

(ii) If $B \subset \stackrel{\circ}{\mathscr{X}}$ is open and convex containing $x$ and if $B$ satisfies (*) above then for all $w \in W$ we have $w B \cap B \neq \varnothing \Leftrightarrow w \in W^{\{x\}}$.

Proof. (i) Let $I_{1}, \ldots, I_{n}$ be closed intervals in linearly independent directions of $V$ lying entirely in $\mathscr{X}$ and containing $x$ in their interiors. Each $I_{j}$ lies inside the union of finitely many facettes (Proposition 8(i)) and each of these facettes intersects $I_{j}$ either in a relatively open subinterval or in a point (since facettes are convex). Let $z_{j_{1}}, \ldots, z_{j k_{(j)}}$ be the points obtained in this fashion. Note that if $H \in \mathbf{H}$ intersects the interior of $I_{j}$ at a single point $p$ then $p=z_{j_{i}}$ for some $1 \leq i \leq k(j)$. Let $J_{j}$ be an open subinterval of $I_{j}$ chosen so that

$$
x \in J_{j} \quad \text { and } \quad z_{j_{i}} \notin J_{j} \text { for all } 1 \leq i \leq k(j) \text { whenever } z_{j_{i}} \neq x .
$$

The above discussion shows that for all $H \in \mathbf{H}$

$$
H \cap J_{j} \neq \varnothing \Leftrightarrow x \in H .
$$

Then the convex hull $B$ of the set $J_{1} \cup \cdots \cup J_{n}$ is by construction a set satisfying $(*)$.

(ii) Let $B \subset \stackrel{\circ}{\mathscr{X}}$ be an open convex neighborhood of $x$ satisfying $(*)$. Assume that $w B \cap B \neq \varnothing$. If $w x \neq x$ then there exists $H \in \mathbf{H}$ separating $x$ and $w x$. By assumption then $B$ and $w B$ lie on opposite sides of $H$. Thus $w B \cap B=\varnothing$. It follows that $w x=x$ and hence that $w \in W^{\{x\}}$.

Next we state without proof equivalent conditions for finiteness of a set of root data. 
Proposition 11. The following are equivalent:

(i) the Cartan matrix $A$ is of finite type;

(ii) there exists $w \in W$ with $w F=-F$;

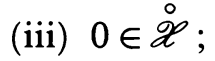

(iv) $\mathscr{Z}=V$;

(v) the number of facettes is finite;

(vi) the number of chambers is finite;

(vii) $W$ is finite;

(viii) $\Sigma$ is finite;

(ix) $\Sigma$ is a finite root system.

The next two results are based on [Ljg] and [MY].

Proposition 12. Let $x \in \mathscr{X}$. Then

$$
x \in \stackrel{\circ}{\mathscr{X}} \Rightarrow W^{\{x\}} \text { is finite. }
$$

In particular the stabilizer of any facette in $\mathscr{\mathscr { C }}$ is finite. If $\Pi$ is finite then

$$
W^{\{x\}} \text { is finite } \Leftrightarrow x \in \mathscr{\mathscr { X }} \text {. }
$$

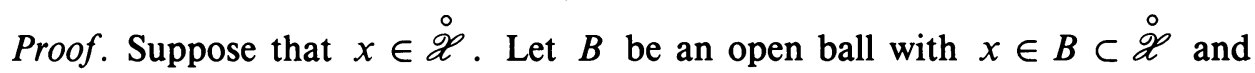
satisfying $(*)$ of Proposition 10 . Any chamber with $x$ in its closure meets $B$ and it follows from Proposition 7 that the set of such chambers is finite. Since $W^{\{x\}}$ permutes these and the action is faithful, $W^{\{x\}}$ is finite.

Now suppose that $\Pi$ is finite and let us prove that $W^{\{x\}}$ finite $\Rightarrow x \in \mathscr{\mathscr { X }}$. We can assume that $x \in F_{\mathbf{I}}$ for some $\mathbf{I} \subset \mathbf{J}$. We let $\Pi_{\mathbf{I}}:=\left\{\alpha_{i} \mid i \in \mathbf{I}\right\}$ and $\Pi_{\mathrm{I}}^{\vee}:=\left\{\alpha_{i}^{\vee} \mid i \in \mathbf{I}\right\}$. Let $A_{\mathbf{I}}:=\left(A_{i j}\right)_{i, j \in \mathbf{I}}$ be the submatrix of $A$ obtained from I. Then

is a set of root data.

$$
\mathscr{D}_{\mathrm{I}}=\left(A_{\mathrm{I}}, \Pi_{\mathrm{I}}, \Pi_{\mathrm{I}}^{\vee}, V, V^{\vee},\langle\cdot, \cdot\rangle\right)
$$

The Weyl group of $D_{\mathbf{I}}$ is the parabolic subgroup $W_{\mathbf{I}}$. For $\mathscr{D}_{\mathbf{I}}$ the fundamental chamber is

$$
F^{(\mathbf{I})}:=\left\{y \in V \mid\left\langle y, \alpha_{i}^{\vee}\right\rangle>0 \text { for all } i \in \mathbf{I}\right\}
$$

and its Tits cone is

$$
\mathscr{X}^{(\mathbf{I})}=\bigcup_{w \in W_{\mathbf{I}}} w \overline{F^{(\mathbf{I})}} .
$$

Suppose that $W^{\{x\}}$ is finite. Since $W^{\{x\}}=W^{F_{1}}=W_{\mathbf{I}}$ (Proposition 12) it follows that $W_{\mathrm{I}}$ is finite. Then by Proposition 11

$$
\mathscr{X}^{(\mathbf{I})}=V \text {. }
$$

Furthermore, since $x \subset F_{\mathrm{I}}$ we have $\left\langle x, \alpha^{\vee}\right\rangle>0$ for all $\alpha^{\vee} \in \Pi^{\vee} \backslash \Pi_{\mathrm{I}}^{\vee}$ and since $W_{\mathbf{I}} x=x,\left\langle x, w \alpha^{\vee}\right\rangle>0$ for all $w \in W_{\mathbf{I}}$ and $\alpha^{\vee} \in \Pi^{\vee} \backslash \Pi_{\mathrm{I}}^{\vee}$. Hence

$$
x \in C:=\left\{y \in V \mid\left\langle y, w \alpha^{\vee}\right\rangle>0 \text { for all } w \in W_{\mathbf{I}}, \alpha^{\vee} \in \Pi^{\vee} \backslash \Pi_{\mathbf{I}}^{\vee}\right\} .
$$


Since $C$ is defined by finitely many inequalities, there exists an open ball $B$ about $x$ lying entirely in $C$.

Let $y \in B$. By $(*)$ and $(* *)$ there is a $w \in W_{\mathrm{I}}$ with $w y \in \overline{F^{(\mathbf{I})}}$. Thus $\left\langle w y, \alpha^{\vee}\right\rangle \geq 0$ for all $\alpha^{\vee} \in \Pi_{\mathrm{I}}^{\vee}$. Since $\left\langle w y, \alpha^{\vee}\right\rangle>0$ for all $\alpha \in \Pi^{\vee} \backslash \Pi_{\mathbf{I}}^{\vee}$ (because $y \in B$ ) we have $w y \in \bar{F} \subset \mathscr{X}$. Thus $y \in \mathscr{X}$ and in conclusion $B \subset \mathscr{X}$ and $x \in \mathscr{\mathscr { X }}$.

Proposition 13. $W$ acts properly discontinuously on $\stackrel{\circ}{\not}$; in other words, given any pair of compact subsets $U$ and $V$ of $\mathscr{\mathscr { X }}$, the set $\{w \in W \mid w U \cap V \neq \varnothing\}$ is finite.

Proof. We may cover each of $U$ and $V$ with a finite number of closed balls $B_{x}$ lying in $\mathscr{\mathscr { X }}$ that satisfy the hyperplane condition $(*)$ of Proposition 10(i): $H \cap B_{x} \neq \varnothing \Rightarrow x \in H$. In particular, by Proposition 7, only finitely many hyperplanes of $\mathscr{H}$ meet each of these balls. It suffices then to prove that if $U$ and $V$ are closed balls and only finitely many hyperplanes of $\mathscr{H}$ meet $U$ and $V$ then the set $\{w \in W \mid w U \cap V \neq \varnothing\}$ is finite. Now in this case one shows that $U$ is covered by finitely many facettes. The same goes for $V$. The relation $w U \cap V \neq \varnothing$ indicates the existence of facettes $\mathscr{C}, \mathscr{C}^{\prime}$ with

$$
\mathscr{C} \cap U \neq \varnothing, \quad \mathscr{C}^{\prime} \cap V \neq \varnothing, \quad w \mathscr{C}=\mathscr{C}^{\prime} .
$$

The same pair $\left(\mathscr{C}, \mathscr{C}^{\prime}\right)$ can occur for only finitely many $w \in W$ since $w^{\prime} \mathscr{C}=$ $\mathscr{C} \Rightarrow w^{\prime} \in W^{\mathscr{E}}$ and $W^{\mathscr{E}}$ is a finite group (by Proposition 12).

\section{SUBROoT SYSTEMS}

Let $\mathscr{D}=\left(A, \Pi, \Pi^{\vee}, V, V^{\vee},\langle\cdot, \cdot\rangle\right)$ be a set of root data over $\mathbf{K}$. In this section we assume that $\operatorname{dim}_{\mathbf{K}} V=\operatorname{dim}_{\mathbf{K}} V^{\vee}=n$ is finite. We maintain all previous notation and terminology for $\mathscr{D}$. Recall that a nonempty subset $\Omega$ of $\Sigma$ is called a subroot system if for all $\alpha, \beta \in \Sigma$

$$
\alpha, \beta \in \Omega \Rightarrow r_{\alpha} \beta \in \Omega .
$$

The main result of this section is

Theorem 6. Let $\mathscr{D}=\left(A, \Pi, \Pi^{\vee}, V, V^{\vee},\langle\cdot, \cdot\rangle\right)$ be a set of root data over $\mathbf{K}$. Let $\Sigma$ be the root system of $\mathscr{D}$ and let $\Omega \subset \Sigma$ be a subroot system. Then there exist a Cartan matrix $B$ and a subset $\Upsilon$ of $\Sigma_{+}$such that if we define $\Upsilon^{\vee}=\left\{\alpha^{\vee} \mid \alpha \in \Upsilon\right\}$ then $\left(B, \Upsilon, \Upsilon^{\vee}, V, V^{\vee},\langle\cdot, \cdot\rangle\right)$ is a set of root data with root system $\Omega$.

The proof of this theorem will be given later as a consequence of a series of preliminary results. Because of the remark about subroot systems made in $\S 4$ it will suffice to establish Theorem 6 in the case when $\mathbf{K}=\mathbf{R}$. We will henceforth assume that this is the case. 
Define an equivalence relation $\sim_{\Omega}$ on $V$ as before but now using the set of hyperplanes $\mathbf{H}_{\Omega}:=\left\{H_{\alpha} \mid \alpha \in \Omega\right\}$. Since $\mathbf{H}_{\Omega} \subset \mathbf{H}$ we have

$$
x \sim_{\mathbf{H}} y \Rightarrow x \sim_{\Omega} y \quad \text { for all } x, y \in V .
$$

The set of chambers relative to $\Omega$ is defined by $\mathscr{C}_{\Omega}:=\{C \mid C$ is an equivalence class of $\sim_{\Omega}$ on $V$ and there exists a nonempty open subset $U$ of $V$ such that

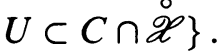

Note. If $F^{\prime}$ is a chamber of $\mathscr{D}$ then $F^{\prime} \subset C$ for some equivalence class $C$ of $\sim_{\Omega}$ on $V$. Clearly $C$ is a chamber relative to $\Omega$. In particular $\mathscr{C}_{\Omega} \neq \varnothing$.

A face of a chamber $C$ is an equivalence class of $\sim_{\Omega}$ on $V$ that lies in $\bar{C}$ and supports a hyperplane $H_{\alpha^{\vee}} \in \mathbf{H}_{\Omega}$. In this case we also say that $H_{\alpha^{\vee}}$ is a wall of $C$. Note that if $\Omega=\Sigma$ then all these concepts coincide with those defined before. The first aim is to prove that walls exist.

Let $W_{\Omega}:=\left\langle r_{\alpha} \mid \alpha \in \Omega\right\rangle$. Since $W_{\Omega}$ stabilizes $\mathbf{H}_{\Omega}$ and $\stackrel{\circ}{\mathscr{D}}$ we see that $W_{\Omega}$ stabilizes $\mathscr{C}_{\Omega}$.

Given $x, y \in \stackrel{\circ}{\mathscr{X}}$ we let

$$
\#_{\Omega}(x, y)=\operatorname{Card}\left\{H \in \mathbf{H}_{\Omega} \mid H \text { separates } x \text { and } y\right\} \text {. }
$$

Note that $\#_{\Omega}(x, y)$ is finite by $\S 5$, Proposition 6 .

Lemma 1. Let $C \in \mathscr{C}_{\Omega}$ and let $x \in C \cap \stackrel{\circ}{\mathscr{X}}$. Let $y \in \grave{\mathscr{X}} \backslash \bigcup_{H \in \mathbf{H}_{\Omega}} H$. Then there exists an open ball $B$ about $y$ in $\stackrel{\circ}{\mathscr{X}}$ such that $\#_{\Omega}(x, y)=\#_{\Omega}(x, z)$ for all $z \in B$.

Proof. Take $B$ to be an open ball about $y$ in $\stackrel{\circ}{\mathscr{X}}$ as in $(*)$ of $\S 5$, Proposition 10. by

Recall that if $x, y \in V, x \neq y$, the (open) ray from $x$ through $y$ is defined

$$
R(x, y):=\left\{x+t(y-x) \mid t \in \mathbf{R}_{>0}\right\} .
$$

Lemma 2. Let $C \in \mathscr{C}_{\Omega}$. Let $x \in C \cap \stackrel{\circ}{\mathscr{X}}$ and $y \in \stackrel{\circ}{\mathcal{X}} \backslash \bar{C}$. If $B$ is any open ball about $y$ in $\stackrel{\mathscr{X}}{\backslash} \bar{C}$ then the cone of rays $\mathscr{R}(x)=\bigcup_{b \in B} R(x, b)$ cuts at least one face of $C$ in an open subset of that face.

Proof. Let $Z \subset B$ be a set of representatives of the lines of $\mathscr{R}(x)$; that is,

(a) $b \in B \Rightarrow \exists z \in Z$ such that $R(x, b)=R(x, z)$ and

(b) $z_{1}, z_{2} \in Z ; z_{1} \neq z_{2} \Rightarrow R\left(x, z_{1}\right) \neq R\left(x, z_{2}\right)$.

If $z \in Z$ the closed interval $[x, z]$ is covered by finitely many classes of $\sim_{\Omega}$ ( $\S 5$, Proposition 8), one of which is $C$ (since $x \in C$ ). Clearly $\bar{C} \cap[x, z]=$ $[x, c(z)]$ for some $c(z) \in[x, y]$. Moreover $c(z) \in \stackrel{\circ}{\mathscr{X}}$ and there exists $\alpha^{\vee}(z) \in$ $\Omega^{\vee}$ such that $c(z) \in H_{\alpha^{\vee}(z)}$. 
For each $\alpha^{\vee} \in \Omega^{\vee}$ let $Z\left(\alpha^{\vee}\right)=\left\{c(z) \mid z \in Z\right.$ and $\left.c(z) \in H_{\alpha^{\vee}}\right\}$. Then there exist $\alpha^{\vee} \in \Omega^{\vee}$ such that $Z\left(\alpha^{\vee}\right)$ spans an affine subspace of dimension $n-1$. (For otherwise $\mathscr{R}$, which is open, would be the union of a countable number of closed subsets of dimension $n-1$, namely

$$
\left.\mathscr{R}=\bigcup_{\alpha^{\vee} \in \Omega^{\vee}}\left(\mathscr{R} \cap\left(\text { affine span of } x \text { and } Z\left(\alpha^{\vee}\right)\right)\right) .\right)
$$

Thus $Z\left(\alpha^{\vee}\right)$ generates $H_{\alpha^{\vee}}$ as an affine space for some $\alpha^{\vee} \in \Omega^{\vee}$. Let $c\left(z_{1}\right)$, $\ldots, c\left(z_{n}\right) \in Z\left(\alpha^{\vee}\right)$ be an affine basis of $H_{\alpha^{\vee}}$. If $S$ denotes the open simplex with the $c\left(z_{i}\right)$ as vertices then $S$ is open in $H_{\alpha^{\vee}}, S \subset H_{\alpha^{\vee}} \cap \stackrel{\circ}{\mathscr{O}}$ and $S \subset \bar{C}$.

Corollary. Walls in $\mathscr{C}_{\Omega}$ exist. Moreover, if $C$ and $C^{\prime}$ are distinct chambers of $\mathscr{C}_{\Omega}$ then there exists a wall separating $C$ and $C^{\prime}$.

Proof. Let $C \in \mathscr{C}_{\Omega}$ (we already know that $\mathscr{C}_{\Omega} \neq \varnothing$ ). It will suffice to show

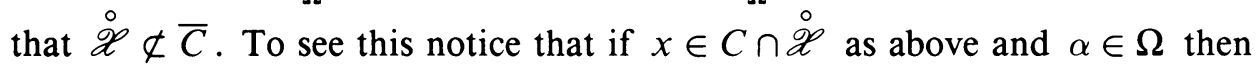
$r_{\alpha} x \in \stackrel{\circ}{\mathscr{X}}$ but $r_{\alpha} x \notin \bar{C}$.

Fix $C \in \mathscr{C}_{\Omega}$. According to the last corollary $C$ has at least one wall. Let $\Upsilon$ be defined as follows:

$$
\Upsilon:=\left\{\alpha \in \Omega \mid H_{\alpha^{\vee}} \text { is a wall of } C \text { and }\left\langle x, \alpha^{\vee}\right\rangle>0 \text { for all } x \in C\right\} \text {. }
$$

Let

$$
W_{\Upsilon}:=\left\langle r_{\alpha} \mid \alpha \in \Upsilon\right\rangle
$$

Proposition 3. Let the notation be as above. Then

(i) $W_{\Upsilon}$ acts transitively ${ }^{2}$ on $\mathscr{C}_{\Omega}$;

(ii) $W_{\Upsilon} \Upsilon=\Omega$ and $W_{\Upsilon} \Upsilon^{\vee}=\Omega^{\vee^{2}}$;

(iii) $\left(\left\langle\alpha, \beta^{\vee}\right\rangle\right)_{\alpha, \beta \in \Upsilon}$ is a Cartan matrix.

Proof. (i) Let $C^{\prime} \in \mathscr{C}_{\Omega}$. It is clear that the cardinal $\#_{\Omega}(x, y)$ with $x \in C$ and $y \in C^{\prime}$ is finite and independent of the choice of $x \in C$ and $y \in C^{\prime}$. We denote this number by $\#\left(C, C^{\prime}\right)$ and reason by induction on it to show that $w C=C^{\prime}$ for some $w \in W_{\Omega}$.

If $\#\left(C, C^{\prime}\right)=0$ then $C=C^{\prime}$ by the last corollary. Assume $N:=\#\left(C, C^{\prime}\right)>$ 0 . Let $U$ be an open subset of $V$ such that $U \subset C^{\prime} \cap \stackrel{\circ}{\mathscr{X}}$. Fix $x \in C \cap \stackrel{\circ}{\mathscr{X}}$. By Lemma 2 we can find $y \in U$ such that the open ray $R(x, y)$ meets a face of $C$. Let $H_{\alpha^{\vee}}, \alpha^{\vee} \in \Upsilon$, be such a face. Let $\left.\left\{x_{1}, \ldots, x_{n}\right\} \in\right] x, y[$ be the distinct points at which a hyperplane of $\mathbf{H}_{\Omega}$ cuts $[x, y]$. We order these points starting from $x$ (see Figure 2). Let $z \in] x_{1}, x_{2}$ [ where $x_{2}:=y$ if $n=1$.

Then $z \in r_{\alpha x} C$ (this is because $H_{\alpha \vee}$ is the unique hyperplane of $\mathbf{H}_{\Omega}$ going through $x_{1}$ since $x_{1}$ lies in a face). Since $\left[r_{\alpha} z, r_{\alpha} y\right]$ is cut by $N-1$ hyperplanes we have $\#\left(C, r_{\alpha} C^{\prime}\right)=N-1$. By induction $r_{\alpha} w C=C^{\prime}$ for some $w \in W_{\Upsilon}$.

2 Once we have established Theorem 6 it will follow from $\S 5$, Proposition 8 (iv) that the action of $W_{\Upsilon}$ on $\mathscr{C}_{\Omega}$ is simply transitive. 


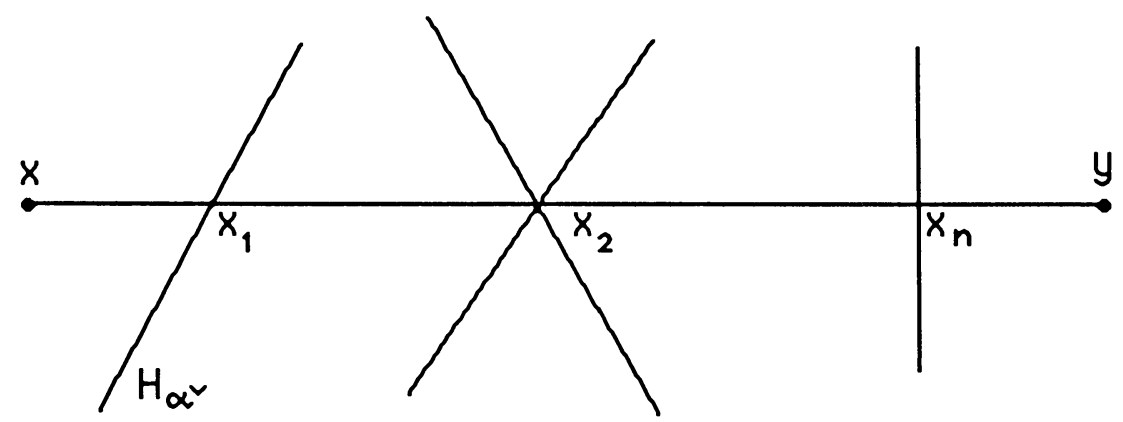

FIGURE 2

(ii) Let $\beta \in \Omega$. Let $x \in H_{\beta^{\vee}} \cap \stackrel{\circ}{\mathscr{X}}$ be chosen so that $x \notin H$ whenever $H \in \mathbf{H}_{\Omega} \backslash\left\{H_{\alpha^{\vee}}\right\}$. By considering a ball around $x$ as in $\S 5$, Proposition 10, we see that $x$ lies on a face of some chamber $C^{\prime}$ relative to $\Omega$ and that $H_{\beta^{\vee}}$ is a wall of this chamber. By (i) we can write $C^{\prime}=w^{-1} C$ for some $w \in W_{\Upsilon}$. Then $w H_{\beta^{\vee}}=H_{w \beta^{\vee}}$ is a wall of $C$ and hence $\pm w \beta^{\vee} \in \Upsilon^{\vee}$. Thus $\beta^{\vee} \in W_{\Upsilon} \Upsilon^{\vee}$. By using the bijection $(\cdot)^{\vee}$ (see $\S 2$ ) we conclude that $\beta \in W_{\Upsilon} \Upsilon=\Omega$.

(iii) Let $\beta \in \Upsilon$. We claim that $H_{\beta^{\vee}}$ is the unique hyperplane of $\mathbf{H}_{\Omega}$ separating $C$ from $r_{\beta} C$. For suppose $H_{\gamma^{\vee}}$ separates these two relative chambers. Then

$$
\left\langle x, \gamma^{\vee}\right\rangle>0 \text { and }\left\langle x, \gamma^{\vee}\right\rangle-\left\langle x, \beta^{\vee}\right\rangle\left\langle\beta, \gamma^{\vee}\right\rangle<0
$$

for all $x \in C$ (we may have to replace $\gamma$ by $-\gamma$ if necessary).

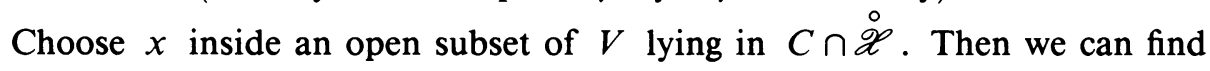
an open ball $B$ about $r_{\beta} x$ with $B \subset r_{\beta} C$ such that the cone of rays from $x$ to this ball $B$ cuts $H_{\beta^{\vee}}$ in an open subset $S$ of $H_{\beta^{\vee}}$. Choose $z \in S$ such that $z \notin H_{\gamma^{\vee}}$. If we now let $x$ approach $z$ in $(*)$ we reach a contradiction. This establishes our claim. In particular, if $\alpha \in \Upsilon, \alpha \neq \beta$, then $H_{\alpha^{\vee}}$ does not separate $C$ and $r_{\beta} C$. Thus

$(* *) \quad\left\langle x, \alpha^{\vee}\right\rangle>0$ and $\left\langle x, \alpha^{\vee}\right\rangle-\left\langle x, \beta^{\vee}\right\rangle\left\langle\beta, \alpha^{\vee}\right\rangle \geq 0$ for all $x \in C$.

If we now let $x$ approach a point of $H_{\alpha^{\vee}}$ not in $H_{\beta^{\vee}}$ we conclude that $\left\langle\beta, \alpha^{\vee}\right\rangle \leq 0$ as desired.

This last proposition contains most of the information needed to establish Theorem 6 . However, we still need a result about lattices to eventually show that RD4 holds.

Lemma 4. Let $L$ be a lattice in $\mathbf{R}^{n}$ and $C$ a convex cone. Suppose that $\stackrel{\circ}{C} \neq \varnothing$. Then $\stackrel{\circ}{C}$ contains a basis of $L$.

Proof. Since $\stackrel{\circ}{C}$ contains balls of arbitrary large diameter (being a cone) we have $L \cap \stackrel{\circ}{C} \neq \varnothing$. Because $C$ is a cone it follows that there exists $v_{1} \in L \cap \stackrel{\circ}{C}$ 
such that $v_{1}$ is primitive. Extend $v_{1}$ to a basis $\left\{v_{1}, v_{2}, \ldots, v_{n}\right\}$ of $L$. For $N$ sufficiently large $N v_{1}+v_{i} \in \stackrel{\circ}{C}$ for all $2 \leq i \leq n$ and it is clear that $\left\{v_{1}, N v_{1}+v_{2}, \ldots, N v_{1}+v_{n}\right\}$ is a basis of $L$.

Lemma 5. Let $L$ be a lattice in $\mathbf{R}^{n}$ and let $C$ be a closed convex cone in $\mathbf{R}^{n}$. Suppose that the dual cone $\mathrm{C}^{+}$has nonempty interior $\stackrel{\circ}{C}^{+}$. For example, this is true $[\mathrm{Rkf}]$ if

(i) $C \cap-C=(0)$ (i.e., $C$ is proper);

(ii) $C$ is defined by a finite number of inequalities (i.e., $C$ is polyhedral).

There exists a basis $\left\{v_{1}, \ldots, v_{n}\right\}$ of $L$ such that the real cone generated by this basis contains $C$.

Proof. Let $L^{*}$ be the $\mathbf{Z}$-dual of $L$. The dual cone to $C$ is

$$
C^{+}:=\left\{x \in \mathbf{R}^{n} \mid\langle x, v\rangle \geq 0 \text { for all } v \in C\right\} .
$$

By assumption $\stackrel{\circ}{C}^{+} \neq \varnothing$. By the previous lemma there exists a basis $\left\{v_{1}^{*}, \ldots\right.$, $\left.v_{n}^{*}\right\}$ of $L^{*}$ contained in $\stackrel{\circ}{C}^{+}$. Let $\Gamma$ be the real cone generated by this basis: $\Gamma=\sum_{i=1}^{n} \mathbf{R}_{\geq 0} v_{i}^{*}$, and let $\Gamma^{+}$be its dual. Then

$$
\Gamma^{+}=\sum_{i=1}^{n} \mathbf{R}_{\geq 0} v_{i}
$$

where $\left\{v_{1}, \ldots, v_{n}\right\}$ is a basis dual to $\left\{v_{1}^{*}, \ldots, v_{n}^{*}\right\}$. Now $\Gamma \subset C^{+} \Rightarrow \Gamma^{+} \supset$ $C^{++}=C$ while $\left\{v_{1}^{*}, \ldots, v_{n}^{*}\right\}$ is a basis of $L^{*} \Rightarrow\left\{v_{1}, \ldots, v_{n}\right\}$ is a basis of $L$.

Proof of Theorem 6. To the subroot system $\Omega$ of $\Sigma$ we have attached the 6-tuple $\left(B, \Upsilon, \Upsilon^{\vee}, V, V^{\vee},\langle\cdot, \cdot\rangle\right)$, which has been shown in Proposition 3 to satisfy RD1-RD3 and is known to have $\Omega$ as its root system. All that remains to be shown is that RD4 holds. Let $\gamma_{1}, \ldots, \gamma_{l}$ be as in RD4 so that

$$
Q=\bigoplus_{i=1}^{l} \mathbf{Z} \gamma_{i} \text { and } \Pi \subset \bigoplus_{i=1}^{l} \mathbf{N} \gamma_{i} \text {. }
$$

Let $C$ be the real cone of $V$ generated by $\left\{\gamma_{1}, \ldots, \gamma_{l}\right\}$. Set

$$
Q^{\prime}=\sum_{\alpha \in \Omega} \mathbf{Z}_{\alpha} \quad(\text { root lattice of } \Omega)
$$

and let $C^{\prime}:=V^{\prime} \cap C$ where $V^{\prime}:=\mathbf{R} \otimes_{\mathbf{Z}} Q^{\prime}$. Note that $C^{\prime}$ is a proper polyhedral cone of $V^{\prime}$ and that $Q^{\prime}$ is a lattice of $V^{\prime}$. By Lemma $5, Q^{\prime}$ contains a basis $\left\{\gamma_{1}^{\prime}, \ldots, \gamma_{m}^{\prime}\right\}$ whose real cone contains $C^{\prime}$. Finally $\Upsilon \subset \Sigma^{+} \subset C$ and hence $\Upsilon \subset C^{\prime}$. Thus

$$
\Upsilon \subset\left(\left(\sum_{i=1}^{m} \mathbf{R}_{\geq 0} \gamma_{i}^{\prime}\right) \cap \bigoplus_{i=1}^{m} \mathbf{Z} \gamma_{i}^{\prime}\right)=\bigoplus_{i=1}^{m} \mathbf{N} \gamma_{i}^{\prime} .
$$

Similar considerations apply to $\Upsilon^{\vee}$. 
Corollary 1 (see also [Ddh]). Let $S \subset \Sigma$ and let $W_{S}=\left\langle r_{\alpha} \mid \alpha \in S\right\rangle$. Then $W_{S}$ is the Weyl group of a set of root data. In particular, $W_{S}$ is a Coxeter group.

Corollary 2 (see also [KW]). Let $\lambda \in V^{\vee}$ and let

$$
\Sigma^{\lambda}:=\{\alpha \in \Sigma \mid\langle\alpha, \lambda\rangle \in \mathbf{Z}\} .
$$

Then $\Sigma^{\lambda}$ is the set of roots of a set of root data. ${ }^{3}$

\section{Conjugacy OF Bases}

Definition. Let

$$
\mathscr{D}=\left(A, \Pi, \Pi^{\vee}, V, V^{\vee},\langle\cdot, \cdot\rangle\right)
$$

be root data with root system $\Sigma$. We assume that $V$ is finite dimensional. A subset $\Upsilon$ of $\Sigma$ is a base of $\Sigma$ if with

$$
B:=\left(\left\langle\alpha, \beta^{\vee}\right\rangle\right)_{\alpha, \beta \in \Upsilon}
$$

we have

$$
\mathscr{D}_{\Upsilon}:=\left(B, \Upsilon, \Upsilon^{\vee}, V, V^{\vee},\langle\cdot, \cdot\rangle\right)
$$

is a set of root data with corresponding root system equal to $\Sigma$.

Thus with the notation of the definition we have

(a) $B$ is a Cartan matrix;

(b) $\Sigma=W_{\Upsilon} \Upsilon$ where $W_{\Upsilon}:=\left\langle r_{\alpha} \mid \alpha \in \Upsilon\right\rangle$;

(c) $V$ admits a fundamental chamber $C$ relative to $\Upsilon$ and $\stackrel{\circ}{C} \neq \varnothing$ whenever the base field is $\mathbf{R}$;

(d) $\Pi$ is a base of $\Sigma$.

It is also clear that

(e) $\Pi^{\vee}$ and $\Upsilon^{\vee}$ are bases of $\Sigma^{\vee}$.

Definition. Let $\Sigma$ be the root system associated with root data $\mathscr{D}$. A subset $S \subset \Sigma$ is decomposable if

$$
S=S_{1} \cup S_{2}, \quad S_{1} \neq \varnothing, S_{2} \neq \varnothing
$$

and for all $\gamma_{1} \in S_{1}, \gamma_{2} \in S_{2}$, we have $\left\langle\gamma_{1}, \gamma_{2}^{\vee}\right\rangle=0 \quad \Leftrightarrow r_{\gamma_{2}} \gamma_{1}=\gamma_{1} \Rightarrow r_{\gamma_{2}} r_{\gamma_{1}}=$ $\left.r_{\gamma_{1}} r_{\gamma_{2}} \Rightarrow\left\langle\gamma_{2}, \gamma_{1}^{\vee}\right\rangle=0\right)$. Otherwise $\Sigma$ is indecomposable.

Let $\mathscr{D}=\left(A, \Pi, \Pi^{\vee}, V, V^{\vee},\langle\cdot, \cdot\rangle\right)$ be a set of root data for $\Sigma$ and let $\Upsilon$ be a base of $\Sigma$.

Proposition 1. $\Pi$ indecomposable $\Leftrightarrow \Sigma$ indecomposable $\Leftrightarrow \Upsilon$ indecomposable. Proof. Suppose that $\Upsilon$ decomposes into $\Upsilon_{1} \cup \Upsilon_{2}$ with $\left\langle\alpha, \beta^{\vee}\right\rangle=0$ for all $\alpha \in \Upsilon_{1}, \beta \in \Upsilon_{2}$. Then with the obvious notation we have $W=W_{\Upsilon_{1}} W_{\Upsilon_{2}}$, the elements of $W_{\Upsilon_{1}}$ and $W_{\Upsilon_{2}}$ commute, $W_{\Upsilon_{1}}\left(\Upsilon_{2}\right)=\Upsilon_{2}, W_{\Upsilon_{2}}\left(\Upsilon_{1}\right)=\Upsilon_{1}$,

$$
(0)=\left\langle W_{\Upsilon_{1}}\left(\Upsilon_{1}\right), \Upsilon_{2}^{\vee}\right\rangle=\left\langle W_{\Upsilon_{1}} W_{\Upsilon_{2}}\left(\Upsilon_{1}\right), \Upsilon_{2}^{\vee}\right\rangle=\left\langle W_{\Upsilon_{1}}\left(\Upsilon_{1}\right), W_{\Upsilon_{2}}\left(\Upsilon_{2}^{\vee}\right)\right\rangle \text {, }
$$

\footnotetext{
${ }^{3}$ We do not know under which conditions $\Sigma^{\lambda}$ admits a finite base.
} 
and

$$
\Sigma=W_{\Upsilon}(\Upsilon)=W_{\Upsilon_{1}}\left(\Upsilon_{1}\right) \cup W_{\Upsilon_{2}}\left(\Upsilon_{2}\right)
$$

is a nontrivial decomposition of $\Sigma$.

Conversely, if $\Sigma$ decomposes as $\Sigma_{1} \cup \Sigma_{2}$ then set $\Upsilon_{i}:=\Upsilon \cap \Sigma_{i}, i=1,2$. If $\Upsilon_{2}=\varnothing$ then $\left\langle\Sigma, \Sigma_{2}^{\vee}\right\rangle=\left\langle W_{\Upsilon_{1}}\left(\Upsilon_{1}\right), \Sigma_{2}^{\vee}\right\rangle=(0)$, which is impossible $\left(\alpha \in \Sigma_{2} \Rightarrow\right.$ $\left.\left\langle\alpha, \alpha^{\vee}\right\rangle \neq 0\right)$.

Since $\Pi$ is a base this argument applies equally well for $\Pi$.

Theorem 2. Let $\mathscr{D}$ be a set of root data as above and let $\Sigma$ be its root system. Assume $\Sigma$ is indecomposable. If $\Upsilon$ and $\Upsilon^{\prime}$ are two bases of $\Sigma$ then there exists an element $w$ of the Weyl group of $\mathscr{D}$ such that either $w \Upsilon=\Upsilon^{\prime}$ or $-w \Upsilon=\Upsilon^{\prime}$.

Corollary. The Cartan matrices of any two bases of an indecomposable root system $\Sigma$ are equal to (up to reindexing the rows/columns). In particular the Cartan matrix is an invariant of $\Sigma$.

Let $\Sigma$ be the root system of $\mathscr{D}$. By an automorphism of $\Sigma$ we shall understand a pair $\left(\sigma, \sigma^{\vee}\right) \in \operatorname{Aut}_{\mathbf{K}}\left(Q_{\mathbf{K}}\right) \times \operatorname{Aut}_{\mathbf{K}}\left(Q_{\mathbf{K}}^{\vee}\right)$ satisfying

Aut 1: $\sigma \Sigma=\Sigma$ and $\sigma^{\vee} \Sigma^{\vee}=\Sigma^{\vee}$,

Aut 2: $(\sigma \alpha)^{\vee}=\sigma^{\vee} \alpha^{\vee}$ for all $\alpha \in \Sigma$,

Aut 3: $\langle x, y\rangle=\left\langle\sigma x, \sigma^{\vee} y\right\rangle$ for all $x \in Q_{\mathbf{K}}$ and $y \in Q_{\mathbf{K}}^{\vee}$.

Let $\operatorname{Aut}(\Sigma)$ denote the group of such automorphisms and identify $W$ inside $\operatorname{Aut}(\Sigma)$ via $w \mapsto\left(w, w^{\vee}\right)$.

Corollary. Let the notation be as above. Then

$$
\begin{aligned}
& W \triangleleft \operatorname{Aut}(\Sigma), \quad W \cap \operatorname{Aut}(\mathscr{D})=\{1\}, \quad \text { and } \\
& \operatorname{Aut}(\Sigma)=W \cdot \operatorname{Aut}(\mathscr{D}) \quad \text { if } \Sigma \text { is finite, } \\
& \operatorname{Aut}(\Sigma)=\{ \pm 1\} \cdot W \cdot \operatorname{Aut}(\mathscr{D}) \quad \text { if } \Sigma \text { is infinite. }
\end{aligned}
$$

Proof of Theorem 2. Using the results of $\S 4$ we can assume that our base field is $\mathbf{R}$. Let $F$ and $C$ be the fundamental chambers for $\Pi$ and $\Upsilon$. It suffices to show that $W C \cap( \pm F) \neq \varnothing$, for in that case, seeing that both $w C$ and $F$ (or $-F$ ) are equivalence classes for $\sim_{\mathbf{H}}, w C= \pm F$ for some $w \in W$. The walls then being identical, $\pm w \Upsilon=\Pi$.

If $A$ is of finite (respectively affine) type, then the interior $\stackrel{\circ}{\mathscr{X}}(\Pi)$ of the Tits

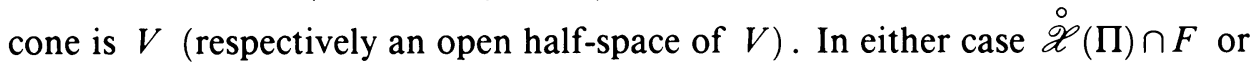

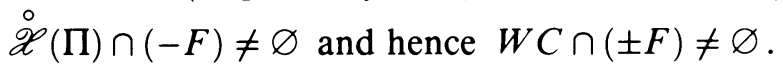

We can suppose then that $A$, and similarly the Cartan matrix $B$ of $\Upsilon$ is of indefinite type. By the last proposition, $A$ and $B$ are indecomposable. Introduce a set $\mathbf{J}_{\Upsilon}$ in 1-1 correspondence with $\Upsilon$ and write

$$
\Upsilon=\left\{\alpha_{i} \mid i \in \mathbf{J}_{\Upsilon}\right\} \text {. }
$$

Let $\widetilde{\mathscr{D}}^{\prime}$ and $\widetilde{\mathscr{D}}$ be sets of universal root data for $B$ and $A$ with corresponding root lattices $\widetilde{Q}^{\prime}$ and $\widetilde{Q}$. Then using the obvious notation there are linear maps 
$\psi^{\prime}, \psi$ for which

$$
\begin{aligned}
& \underset{\tilde{Q}_{\mathbf{R}}^{\prime}}{\stackrel{\psi^{\prime}}{\rightarrow}} \quad Q_{\mathbf{R}} \stackrel{\psi}{\longleftarrow} \quad \widetilde{Q}_{\mathbf{R}} \\
& \tilde{\Upsilon} \cong \Upsilon \\
& \Pi \stackrel{\Upsilon}{ } \simeq \widetilde{\Pi}
\end{aligned}
$$

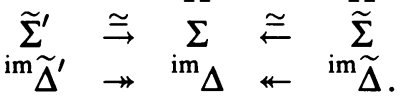

Choose $\mathbf{I} \subset \mathbf{J}_{\Upsilon}$ with the following properties:

(a) $\mathbf{I}$ is finite and $B^{\mathbf{I}}:=\left(B_{i j}\right)_{i, j \in \mathbf{I}}$ is indecomposable;

(b) $B^{I}$ is indefinite;

(c) $\Upsilon_{\mathbf{I}}:=\left\{\alpha_{i} \mid i \in \mathbf{I}\right\}$ spans $Q_{\mathbf{R}}$

(although $\widetilde{Q}_{\mathbf{R}}^{\prime}$ and $\widetilde{Q}_{\mathbf{R}}$ may be infinite dimensional, $Q_{\mathbf{R}}$ is finite dimensional). Since $B^{\mathbf{I}}$ is indecomposable and indefinite there is an imaginary root $\tilde{\gamma} \in$ ${ }^{i m} \widetilde{\Delta}_{+}^{\prime}$ (positive relative to $\widetilde{\Upsilon}$ ) such that

(i) $\operatorname{supp}\left(\tilde{\gamma}^{\prime}\right)=\mathbf{I}$;

(ii) $\left\langle\tilde{\gamma}^{\prime}, \tilde{\alpha}_{j}^{\prime \vee}\right\rangle<0$ for all $j \in \mathbf{I}$ (see [Kac]).

Let $\psi^{\prime}\left(\tilde{\gamma}^{\prime}\right)=: \gamma \in{ }^{\mathrm{im}} \Delta$ and choose $\tilde{\gamma} \in{ }^{\mathrm{im}} \widetilde{\Delta}$ with $\psi(\tilde{\gamma})=\gamma$.

Now

$$
\left\langle\tilde{\gamma}^{\prime}, \alpha_{j}^{\prime \vee}\right\rangle \leq 0 \text { for all } j \in \mathbf{J}_{\Upsilon}
$$

(by construction and because $\operatorname{supp}\left(\tilde{\gamma}^{\prime}\right)=\mathbf{I}$ ) and hence

$$
\left\langle\gamma, \alpha_{j}^{\vee}\right\rangle \leq 0 \text { for all } j \in \mathbf{J}_{\Upsilon}
$$

with strict inequality on $\mathbf{I}$. However, we claim that

$$
\left\langle\gamma, \alpha_{j}^{\vee}\right\rangle<0 \text { for all } j \in \mathbf{J}_{\Upsilon} \text {. }
$$

For suppose that $\left\langle\gamma, \alpha_{j}^{\vee}\right\rangle=0$ for some $j\left(\in \mathbf{J}_{\Upsilon} \backslash \mathbf{I}\right)$. Write

$$
\tilde{\gamma}^{\prime}=\sum_{i \in \mathbf{I}} c_{i} \tilde{\alpha}_{i}^{\prime}, \quad c_{i}>0
$$

Then

$$
\gamma=\sum_{i \in \mathbf{I}} c_{i} \alpha_{i}
$$

and

$$
0=\left\langle\gamma, \alpha_{j}^{\vee}\right\rangle=\sum_{i \in \mathbf{I}} c_{i}\left\langle\alpha_{i}, \alpha_{j}^{\vee}\right\rangle \Rightarrow\left\langle\alpha_{i}, \alpha_{j}^{\vee}\right\rangle=0 \quad \text { for all } i \in \mathbf{I} \text {. }
$$

But $\alpha_{j}$ lies in the span of $\Upsilon_{\mathbf{I}}$ so $2=\left\langle\alpha_{j}, \alpha_{j}^{\vee}\right\rangle=0$. This contradiction shows that $-\gamma \in C$.

Since no hyperplane $H_{\alpha}$ meets $C$,

$$
\left\langle\gamma, \alpha^{\vee}\right\rangle \neq 0 \text { for all } \alpha \in \Sigma \text {. }
$$

Now $\tilde{\gamma} \in{ }^{\mathrm{im}} \tilde{\Delta}_{ \pm}$(relative to $\left.\widetilde{\Pi}\right)$. Suppose that $\tilde{\gamma} \in{ }^{\mathrm{im}} \tilde{\Delta}_{+}$. Choose $w \in W$ so that $\mathrm{ht}_{\widetilde{\Pi}}(w \tilde{\gamma})$ is minimal and therefore

$$
\left\langle w \tilde{\gamma}, \tilde{\alpha}^{\vee}\right\rangle \leq 0 \text { for all } \tilde{\alpha} \in \tilde{\Pi} .
$$


Then

$$
\begin{array}{rlr}
(*) & \Rightarrow\left\langle w \tilde{\gamma}, \tilde{\alpha}^{\vee}\right\rangle<0 & \text { for all } \tilde{\alpha} \in \widetilde{\Pi} \\
& \Rightarrow\left\langle w \gamma, \alpha^{\vee}\right\rangle<0 & \text { for all } \alpha \in \Pi \\
& \Rightarrow-w \gamma \in F . &
\end{array}
$$

Thus $-w \gamma \in F \cap w C$. If $\tilde{\gamma} \in{ }^{\mathrm{im}} \tilde{\Delta}_{-}$we conclude similarly that $w \gamma \in-w C \cap F$. This is what we wanted to prove.

Finally, there is no $w \in W$ with $w F=-F$ (in the nonfinite case) since then $w \Sigma_{+}=\Sigma_{-}$, contradicting the finiteness of the length function $l(w)$.

Remark. Maxwell's Demon shows that $\operatorname{Aut}(\Upsilon)$ can be infinite. More precisely, one can show that for the $\operatorname{Demon} \operatorname{Aut}(\Upsilon) \simeq \operatorname{Aut}(\mathscr{D}) / \pm W$ is infinite dihedral.

We finish this section by describing an algorithm for computing bases of subroot systems. Let $\mathscr{D}=\left(A, \Pi, \Pi^{\vee}, V, V^{\vee},\langle\cdot, \cdot\rangle\right)$ be a set of root data. Let $\Sigma$ be its root system and let $\Omega$ be a subroot system of $\Sigma$. Let $\beta_{1}, \beta_{2}, \ldots$ be a total ordering of $\Omega \cap \Sigma_{+}$in such a way as to respect increasing height (relative to the natural expression in terms of $\Pi$ ). A base $\Upsilon$ of $\Omega$ can be found by setting

(i) $\beta_{1} \in \Upsilon$,

(ii) if $n>1$ then $\beta_{n} \notin \Upsilon$ if and only if

$$
\beta_{n} \cap\left(\sum_{i=1}^{n-1} \mathbf{N} \beta_{i}\right) \neq(0) \text {. }
$$

In other words, $\Upsilon$ is constructed so as to satisfy WIP.

\section{Relations With Lie algebras}

Let $\mathscr{D}=\left(A, \Pi, \Pi^{\vee}, V, V^{\vee},\langle\cdot, \cdot\rangle\right)$ be a set of root data over $\mathbf{K}$ with associated (real) root system $\Sigma$. We construct a Kac-Moody-like Lie algebra over the $\mathbf{K}$ as follows: form the free Lie algebra $\mathfrak{e}$ on generators $e_{i}, f_{i}, i \in \mathbf{J}$, and form the free product $V^{\vee} * \mathfrak{e}$ where $V^{\vee}$ is given an abelian Lie algebra structure. Now factor out the well-known relations for all $i, j \in \mathbf{J}$ and for all $h \in V^{\vee}$

$$
\begin{aligned}
{\left[e_{i}, f_{j}\right] } & =\delta_{i j} \alpha_{j}^{\vee}, \\
{\left[h, e_{j}\right] } & =\left\langle\alpha_{j}, h\right\rangle e_{j}, \\
{\left[h, f_{j}\right] } & =-\left\langle\alpha_{j}, h\right\rangle f_{i}, \\
\left(\operatorname{ad} e_{i}\right)^{-A_{j i}+1} e_{j}=0 & =\left(\operatorname{ad} f_{i}\right)^{-A_{j i}+1} f_{j} \quad \text { if } i \neq j .
\end{aligned}
$$

We denote this Lie algebra by $\mathfrak{g}(\mathscr{D})$. It differs somewhat from the usual definition of a Kac-Moody algebra inasmuch as $\left\{\alpha_{i} \mid i \in \mathbf{J}\right\}$ is not required to be linearly independent in $V$. Otherwise $\mathfrak{g}=\mathfrak{g}(\mathscr{D})$ has the usual root space decomposition

$$
\mathfrak{g}=\bigoplus_{\alpha \in \Sigma^{*}} \mathfrak{g}^{\alpha}, \quad \mathfrak{g}^{0}=V^{\vee}
$$

where $\Sigma^{*}$ is the root string closure of $\Sigma$. The real roots of $\mathfrak{g}$ relative to $V^{\vee}$ are precisely the elements of $\Sigma$. 
Following the conventional definition from finite-dimensional theory, we have

Definition. Let $\mathscr{D}$ be root data with root system $\Sigma$ and let $\mathscr{D}^{\prime}$ be a set of subroot data with root system $\Sigma^{\prime}$. We say that $\mathscr{D}^{\prime}$ is closed in $\mathscr{D}$ and $\Sigma^{\prime}$ is closed in $\Sigma$ if

$$
\alpha, \beta \in \Sigma^{\prime}, \alpha+\beta \in \Sigma \Rightarrow \alpha+\beta \in \Sigma^{\prime} .
$$

Proposition 1. Let $\mathscr{D}$ be a set of root data with root system $\Sigma$ and let $\mathscr{D}^{\prime}$ be a set of subroot data of $\mathscr{D}$ with root system $\Sigma^{\prime}$. The following are equivalent.

(i) $\mathscr{D}^{\prime}$ is closed in $\mathscr{D}$.

(ii) If $\Upsilon$ is any base of $\Sigma^{\prime}$ then $\forall \alpha, \beta \in \Upsilon, \alpha-\beta \notin \Sigma$.

(iii) The subalgebra of $\mathfrak{g}(\mathscr{D})$ generated by $V^{\vee}$ and the root spaces $\left\{\mathfrak{g}^{\alpha} \mid \alpha \in\right.$ $\pm \Upsilon\}$, where $\Upsilon$ is any base of $\Sigma^{\prime}$, is a homomorphic image of $\mathfrak{g}\left(\mathscr{D}^{\prime}\right)$ by a homomorphism that preserves root spaces and maps $V^{\vee} \subset \mathfrak{g}\left(\mathscr{D}^{\prime}\right)$ identically onto itself in $\mathfrak{f}$.

Proof. (i) $\Rightarrow$ (ii) Let $\Upsilon$ be a base of $\Sigma^{\prime}$ and suppose that $\alpha, \beta \in \Upsilon$ and $\alpha-\beta \in$ $\Sigma$. Then $r_{\beta}(\alpha-\beta)=r_{\beta} \alpha+\beta \in \Sigma$. Since $r_{\beta} \alpha, \beta \in \Sigma^{\prime}$ so does $r_{\beta} \alpha+\beta$ by assumption. Thus also $\alpha-\beta \in \Sigma^{\prime}$, which violates WIP.

(ii) $\Rightarrow$ (iii) Let $\mathfrak{g}=\mathfrak{g}(\mathscr{D})$. For each $\alpha \in \Upsilon, \pm \alpha \in \Sigma$ so $\mathfrak{g}^{\alpha}+\mathbf{K} \alpha^{\vee}+\mathfrak{g}^{-\alpha}$ is isomorphic to $\mathfrak{s l}_{2}(\mathbf{K})$. Let $e_{\alpha} \in \mathfrak{g}^{\alpha}, f_{\alpha} \in \mathfrak{g}^{-\alpha}$ with $\left[e_{\alpha}, f_{\alpha}\right]=\alpha^{\vee}$. Given $\alpha, \beta \in \Upsilon, \alpha \neq \beta$, let us show that $\alpha-\beta \notin \Delta$ (namely the root string closure of $\Sigma$ ). Otherwise $\gamma:=\alpha-\beta \in \Delta \backslash \Sigma$ so that $\Sigma$ is not of finite type. By $\S 3$, Proposition 3, we can choose $\tilde{\gamma} \in \tilde{\Delta} \mid \widetilde{\Sigma}$ such that $\tilde{\gamma} \rightarrow \gamma$. We may assume that $\tilde{\gamma} \in \tilde{\Delta}_{-}$. Choose $w \in \widetilde{W}$ such that $w \tilde{\gamma}$ satisfies $\left\langle w \tilde{\gamma}, \tilde{\alpha}_{j}^{\vee}\right\rangle \geq 0$ for all $j \in \mathbf{J}$. Then by $\S 2$, Proposition 3, we have $\left\langle w \gamma, \alpha_{j}^{\vee}\right\rangle \geq 0$ for all $j \in \mathbf{J}$. Thus $w \gamma$ is in the closure of the fundamental chamber and hence $\gamma$ lies in the Tits cone $\mathscr{X}$. Now $r_{\beta}(\alpha-\beta)=\alpha+n \beta \in \mathscr{X}$ with $n=-\left\langle\alpha, \beta^{\vee}\right\rangle+1>0$ since $\alpha, \beta \in \Upsilon$. Thus $\alpha-\beta$ and $\alpha+n \beta$ both in $\mathscr{Z}$ implies that $\alpha \in \mathscr{Z}$ by convexity. Thus $w \alpha$ is dominant for some $w \in W$ and therefore $\Sigma$ is of finite type, contrary to assumption. (This argument assumes that $k=\mathbf{R}$ and $\Sigma$ is indecomposable. We leave to the reader to check that this assumption can be made without any loss of generality.)

Having established that $\alpha-\beta \notin \Delta$ we have

$$
\left[e_{\alpha}, f_{\beta}\right]=\delta_{\alpha \beta} \alpha^{\vee} \text {. }
$$

The identities $\left[h, e_{\alpha}\right]=\langle\alpha, h\rangle e_{\alpha},\left[h, f_{\alpha}\right]=-\langle\alpha, h\rangle f_{\alpha}$ for all $\alpha \in \Sigma, h \in V^{\vee}$ are obvious. Since ad $e_{\alpha}$ is locally nilpotent in $\mathfrak{g}$ and since $\left[f_{\alpha}, e_{\beta}\right]=0$ if $\alpha \neq \beta$, it follows from $\mathfrak{s l}_{2}$-theory that

$$
\left(\operatorname{ad} e_{\alpha}\right)^{-\left\langle\beta, \alpha^{\vee}\right\rangle+1} e_{\beta}=0
$$

and likewise that

$$
\left(\operatorname{ad} f_{\alpha}\right)^{-\left\langle\beta, \alpha^{\vee}\right\rangle+1} f_{\beta}=0 .
$$


Thus there is a surjective homomorphism

$$
\mathfrak{g}\left(\mathscr{D}^{\prime}\right) \rightarrow \mathfrak{f}
$$

that is the identity map on $V^{\vee}$ and maps root spaces onto root spaces.

(iii) $\Rightarrow$ (i) Let $\alpha, \beta \in \Sigma^{\prime}$ and suppose that $\alpha+\beta \in \Sigma$. Then in $\mathfrak{g}(\mathscr{D})$ the $\alpha$-string through $\beta$ contains $\alpha+\beta$, so $\left[\mathfrak{g}^{\alpha}, \mathfrak{g}^{\beta}\right] \neq(0)$. Since $\mathfrak{g}^{\alpha}, \mathfrak{g}^{\beta} \subset \mathfrak{f}$, the corresponding root spaces $\mathfrak{g}^{\prime \alpha}$ and $\mathfrak{g}^{\prime \beta}$ in $\mathfrak{g}\left(\mathscr{D}^{\prime}\right)$ satisfy $\left[\mathfrak{g}^{\prime \alpha}, \mathfrak{g}^{\prime \beta}\right] \neq(0)$ and hence $\alpha+\beta$ is a root of $\mathfrak{g}\left(\mathscr{D}^{\prime}\right)$. Thus either $\alpha+\beta \in \Sigma^{\prime}$, which is what we want, or $\alpha+\beta$ is an imaginary root in the string closure $\Delta^{\prime}$ of $\Sigma^{\prime}$. But then $2(\alpha+\beta) \in \Delta^{\prime}$ and hence $2(\alpha+\beta) \in \Delta$. This is impossible since $\alpha+\beta \in \Sigma$.

Conjecture. The homomorphism in part (iii) of the proposition is an isomorphism. It is evident that the kernel $\mathfrak{a}$ of this map is a $Q$-homogeneous ideal of $\mathfrak{g}\left(\mathscr{D}^{\prime}\right)$ which intersects $V^{\vee}$ trivially; that is, $\mathfrak{a}$ is a radical ideal. Thus in the symmetrizable case $\mathfrak{a}=(0)$ and we indeed have an isomorphism. In the case that $\Upsilon$ is a subset of $\Pi$ it is an isomorphism in any case [MP].

Corollary. Let $\Sigma^{\prime} \subset \Sigma$ be a closed subroot system of the root system $\Sigma$. Then the subalgebra of $\mathfrak{g}(\mathscr{D})$ generated by the root spaces $\mathfrak{g}^{\alpha}, \alpha \in \Sigma^{\prime}$, is a homomorphic image of $\mathfrak{g}\left(\mathscr{D}^{\prime}\right)$ by a homomorphism which preserves root spaces and maps $V^{\vee}$ identically onto itself. This is an isomorphism if $A$ is symmetrizable.

Example 6. Consider Examples 1 and 3. The set of subroot data $\mathscr{D}^{\prime}$ is closed in $\mathscr{D}$ and $\Omega$ is a closed subroot system of $\Sigma$. This is easily checked by applying Proposition 1(ii) to $\Upsilon$. Thus in the rank 3 hyperbolic Lie algebra $\mathfrak{g}(\mathscr{D})$, the subalgebra generated by the root spaces $\mathfrak{g}^{\alpha}, \alpha \in \Omega$, is an isomorphic image of $\mathfrak{g}\left(\mathscr{D}^{\prime}\right)$. A comparison of $\mathfrak{g}\left(\mathscr{D}^{\prime}\right)$ and the usual Kac-Moody Lie algebra $\mathfrak{g}(B, R)$ defined by a realization of the Cartan matrix $B$ is interesting. The Lie algebra $\mathfrak{g}(B, R)$ has an infinite-dimensional diagonal subalgebra $\mathfrak{h}$ which includes $\bigoplus_{\alpha \in \Upsilon^{\prime}}, \mathbf{K} \alpha^{\vee}$ where $\Upsilon^{\prime}$ is a set in 1-1 correspondence with $\Upsilon$. Since $B$ has row rank $3, \mathfrak{h}$ has additional elements that serve to make the elements of $\Upsilon$ appear as linearly independent functionals on $\mathfrak{h}$. The derived algebra $\mathfrak{g}^{\prime}(B, R)$ has diagonal algebra $\bigoplus_{\alpha \in \Upsilon^{\prime}}, \mathbf{K} \alpha^{\vee}$. There is an obvious homomorphism $\psi$ of $\mathfrak{g}^{\prime}(B, R)$ onto $\mathfrak{g}(\mathscr{D})$ in which $\bigoplus_{\alpha \in \Upsilon^{\prime}} \mathbf{K} \alpha^{\vee} \rightarrow V^{\vee}$. The kernel of this map is a codimension-3 ideal in $\bigoplus_{\alpha \in \Upsilon^{\prime}} \mathbf{K}_{\alpha} \mathrm{V}^{\alpha}$ which is in fact the center of $\mathfrak{g}^{\prime}(B, R)$. In the first place the radical of $\mathfrak{g}^{\prime}(B, R)$ is $(0)$ and hence the kernel of $\psi$ lies in $\bigoplus_{\alpha \in \Upsilon^{\prime}} \mathbf{K}_{\alpha}^{\vee}$. Then it is obvious that $\operatorname{ker} \psi$ must be central. But the center of $\mathfrak{g}\left(\mathscr{D}^{\prime}\right)$ is $(0)$ since the row rank of $B=3=\operatorname{dim} V^{\vee}$.

Example 7 (The Leech lattice and the Monster Lie algebra). The reader is referred to [CS, Chapters 28 and 30] for more details.

The unique unimodular lattice of signature $\left(1^{25},-1\right)$ in 26-dimensional real space $V$ is

$$
\Lambda^{25,1}:=\left\{x=\left(x_{0}, \ldots, x_{24} ; x_{25}\right) \in \mathbf{Z}^{26} \cup\left(\mathbf{Z}+\frac{1}{2}\right)^{26} \mid \sum_{i=0}^{24} x_{i}-x_{25} \in 2 \mathbf{Z}\right\}
$$


with associated symmetric bilinear form

$$
(x \mid x)=x_{0}^{2}+\cdots+x_{24}^{2}-x_{25}^{2} .
$$

Let

$$
w:=(0,1,2, \ldots, 24 ; 70),
$$

which is isotropic, and define the set of Leech roots

$$
\Pi:=\left\{x \in \Lambda^{25,1} \mid(x \mid x)=2,(x \mid w)=-1\right\} .
$$

The Leech roots form a set which is in natural 1-1 correspondence with the Leech lattice. Furthermore

$$
L:=\{(x \mid y) \mid x, y \in \Pi\}
$$

is a Cartan matrix (indexed by the infinite set $\Pi$ ) .

We claim that

$$
\mathscr{M}:=(L, \Pi, \Pi, V, V,(\cdot \mid \cdot))
$$

is a set of root data. Axioms RD1-RD3 obviously hold. We sketch how to prove RD4. First, the Leech roots generate $\Lambda^{25,1}$ as a lattice. This is easily seen from the list of Leech roots in Chapter 28 of [CS]. We have to find a basis for $\Lambda^{25,1}$ that contains $\Pi$ in its positive cone. We use the method of $\S 6$, Theorem 1. According to [CS], if $\Pi_{k}:=\left\{x \in \Pi \mid x_{25}=k\right\}$ then $k \geq 0$ and $\Pi_{0}$ is a base for a root system of type $D_{25}$. Let - denote the usual scalar product in $\mathbf{R}^{26}$ (coordinates indexed from 0 to 25 ) and let $e_{25}$ be a unit vector along the $x_{25}$ axis. The closed convex cone

$$
C=\left\{\begin{array}{l|l}
x \in \mathbf{R}^{26} & \frac{x \cdot e_{25}}{(x \mid x)^{1 / 2}} \geq \frac{1}{\sqrt{2}}
\end{array}\right\}
$$

contains all $x \in \mathbf{R}^{26}$ with $x_{25} \geq 1$ and $(x \mid x) \leq 2$. In particular $C \supset \bigcup_{k \geq 1} \Pi_{k}$. The dual cone $C^{+}$of $C$ has nonempty interior.

Let $\mathbf{R}^{25}$ denote the subspace $x_{25}=0$ of $\mathbf{R}^{26}$ and let $E$ be a proper closed polyhedral cone in $\mathbf{R}^{25}$ containing $\Pi_{0}$ (it exists because $\Pi_{0}$ is a base for $D_{25}$ ). Let $\widetilde{E}=E \times \mathbf{R}_{\geq 0}$ be the prism above $E$. Then the dual $\widetilde{E}^{+}$of $\widetilde{E}$ is $E^{+} \times \mathbf{R}_{\geq 0}$ where $E^{+}$is the dual of $E$. It is clear that $U:=\left(C^{+}\right)^{\circ} \cap\left(\widetilde{E}^{+}\right)^{\circ} \neq \varnothing$ since $\left(C^{+}\right)^{\circ}$ is completely symmetric with respect to the $x_{25}$-axis. Thus $U$ contains a basis $B=\left\{u_{0}^{0}, \ldots, u_{25}^{0}\right\}$ for the dual lattice $\Lambda_{25,1}^{0}$ (relative to $\cdot$ ). Then a basis $\left\{u_{0}, \ldots, u_{25}\right\}$ of $\Lambda_{25,1}$ dual to $B$ has the property $\sum \mathbf{R}_{\geq 0} u_{i} \supset C \cup E \supset \Pi$ so $\Pi \subset \sum_{i=0}^{25} \mathbf{Z}_{\geq 0} u_{i}$. This proves RD4. We have $\operatorname{Aut}(\Pi) \simeq \mathrm{Co}_{\infty}$ (the Conway group extended by all translations of the Leech lattice).

The resulting Lie algebra $\mathfrak{g}(\mathscr{M})$ is the Monster Lie algebra of [CS, Chapter 30] (already reduced so that the diagonal subalgebra is of dimension 26). 


\section{REFERENCES}

[Bbk] N. Bourbaki, Groupes et algèbres de Lie, Chapters 4-6, Hermann, Paris, 1968.

[Ctd] C. Caratheodory, Theory of functions, vol. 2, Chelsea, New York, 1960.

[Ctr] R. W. Carter, Simple groups of Lie type, Wiley, New York, 1972.

[CS] J. H. Conway and N. J. A. Sloane, Sphere packings, lattices and groups, Grundlehren Math. Wiss., 290, Springer, Berlin, 1988.

[Ddh] V. Deodhar, A note on subgroups generated by reflections in Coxeter groups (to appear).

[Kac] V. Kac, Infinite dimensional Lie algebras, Cambridge University Press, 1985.

[KW] V. Kac and M. Wakimoto, Modular invariant representations of infinite dimensional Lie algebras and superalgebras, preprint.

[Ljg] E. Looijenga, Invariant theory for generalized root systems, Invent. Math. 61 (1980), 1-32.

[Mdy] R. V. Moody, Root systems of hyperbolic type, Adv. Math. 33 (1979), 144-160.

[MP] R. V. Moody and A. Pianzola, Lie algebras with triangular decomposition, vol. 1, CMS Series, Wiley, New York (to appear).

[MY] R. V. Moody and T. Yokonuma, Root systems and Cartan matrices, Canad. J. Math. 34 (1982), 63-79.

[Rkf] R. T. Rockafellar, Convex analysis, Princeton Math. Ser., vol. 28, Princeton University Press, Princeton, N.J., 1970.

[Stn] R. Steinberg, Endomorphisms of linear algebraic groups, Mem. Amer. Math. Soc. No. 80, (1968).

[Vnb] E. B. Vinberg, Some arithmetical discrete groups in Lobaĉevskii spaces, Discrete Subgroups of Lie Groups, Oxford University Press, London/New York, 1975, pp. 323-348.

Department of Mathematics, University of Saskatchewan, Saskatoon, SaskatcheWAN, CANADA S7N 0W0

Department of Mathematics, University of Alberta, Edmonton, Alberta, Canada T6G 2G1 (Current address of both authors) 